\title{
ON A THEOREM OF KUCEROVSKY FOR HALF-CLOSED CHAINS
}

\author{
JENS KAAD AND WALTER D. VAN SUIJLEKOM
}

\begin{abstract}
Kucerovsky's theorem provides a method for recognizing the interior Kasparov product of selfadjoint unbounded cycles. In this paper we extend Kucerovsky's theorem to the nonselfadjoint setting by replacing unbounded Kasparov modules with Hilsum's half-closed chains. On our way we show that any halfclosed chain gives rise to a multitude of twisted selfadjoint unbounded cycles via a localization procedure. These unbounded modular cycles allow us to provide verifiable criteria avoiding any reference to domains of adjoints of symmetric unbounded operators.
\end{abstract}

\section{INTRODUCTION}

In recent years a lot of attention has been given to the non-unital framework for noncommutative geometry, where the absence of a unit is interpreted as a non-compactness condition on the underlying noncommutative space, CON94, LAT13, CGRS14, MERE16]. For a more detailed analysis of the non-compact setting it is important to distinguish between the complete and the non-complete case, MERE16. Whereas the complete case is still modelled by a (non-unital) spectral triple or more generally an unbounded Kasparov module, the lack of completeness leads to the non-selfadjointness of symmetric differential operators. A noncommutative geometric framework that captures the non-complete setting is provided by Hilsum's notion of a halfclosed chain, where the selfadjointness condition on the unbounded operator is replaced by a more flexible symmetry condition, HIL10. This framework is supported by results of Baum, Douglas, Taylor and Hilsum showing that any first-order symmetric elliptic differential operator on any Riemannian manifold gives rise to a half-closed chain, BDT89, HiL10.

Unbounded Kasparov modules give rises to classes in Kasparov's KK-theory via the Baaj-Julg bounded transform and this result has been extended by Hilsum to cover half-closed chains, [BAJU83, HIL10].

Date: November 13, 2018.

2010 Mathematics Subject Classification. 19K35; 58B34.

Key words and phrases. Unbounded Kasparov modules, Half-closed chains, Unbounded modular cycles, $K K$-theory, Unbounded $K K$-theory, Kasparov product, Unbounded Kasparov product. 
This transform contains information about the algebraic topology of the original geometric situation described by a half-closed chain.

The main structural property of Kasparov's KK-theory is the interior Kasparov product, KAS80:

$$
\widehat{\otimes}_{B}: K K(A, B) \times K K(B, C) \rightarrow K K(A, C) .
$$

The interior Kasparov product is however not explicitly constructed and it is therefore important to develop tools for computing the interior Kasparov product of two given Kasparov modules. Given three classes in KK-theory, Connes and Skandalis developed suitable conditions for verifying whether one of these three classes factorizes as an interior Kasparov product of the remaining two classes, COSK84.

The conditions of Connes and Skandalis were translated to the unbounded setting by Kucerovsky, [KUC97. Thus, given three unbounded Kasparov modules, Kucerovsky's theorem provides criteria for verifying that one of these unbounded Kasparov modules factorizes as an unbounded Kasparov product of the remaining two unbounded Kasparov modules. In many cases, the conditions are easier to verify directly at the unbounded level, using Kucerovsky's theorem, instead of first applying the bounded transform and then relying on the results of Connes and Skandalis. Indeed, in the unbounded setting we are usually working with first-order differential operators whereas their bounded transforms are zeroth-order pseudo-differential operators involving a square root of the resolvent.

In this paper we extend Kucerovsky's theorem to cover the noncomplete setting, where the unbounded Kasparov modules are replaced by half-closed chains. The main challenge in carrying out such a task is that the domain of the adjoint of a symmetric unbounded operator can be difficult to describe. The original proof of Kucerovsky does therefore not translate to the non-selfadjoint setting as the correct conditions have to be formulated without any reference to maximal domains of symmetric unbounded operators.

The main technique that we apply is a localization procedure relating to the work of the first author in [KAA15, KAA17. This procedure allows us to pass from a symmetric regular unbounded operator $D$ to an essentially selfadjoint regular unbounded operator of the form $x D x^{*}$ for an appropriate bounded adjointable operator $x$. In the case where $D$ is a Dirac operator, the localization corresponds to a combination of two operations: restricting all data to an open subset and passing from the non-complete Riemannian metric on this open subset to a conformally equivalent but complete Riemannian metric. The size of the open neighborhood and the relevant conformal factor are both determined by the positive function $x x^{*}$.

In particular, our technique allows us to construct a multitude of unbounded modular cycles out of a given half-closed chain. We interpret 
this localization procedure in terms of the unbounded Kasparov product by the module generated by the localizing element $x$. In this way, we may work with selfadjoint unbounded operators and hence eliminate the difficulties relating to the description of maximal domains. On the other hand, the "conformal factor" $\left(x x^{*}\right)^{-2}$ produces a twist of the commutator condition and this twist is described by the modular automorphism $\sigma(\cdot)=\left(x x^{*}\right)(\cdot)\left(x x^{*}\right)^{-1}$. We refer to Connes and Moscovici for further discussion of this issue in the case where $x$ is positive and invertible, see CoMo08].

The present paper is motivated by the geometric setting of a proper Riemannian submersion of $\operatorname{spin}^{c}$-manifolds, and the criteria that we develop here have already been applied in [KAVS17] to obtain factorization results involving the corresponding fundamental classes in KK-theory.

Our results may also be of importance for the further development of the unbounded Kasparov product as initiated by Connes in CON96 and developed further by Mesland and others in [MEs14, KALE13, BMvS16, KAA15, MERE16, KAA16.

The structure of this paper is as follows: In Section 2 and Section 3 we review the concept of a half-closed chain and of an unbounded modular cycle. In Section 4, Section 5 and Section 6 we prove our results on the localization procedure and investigate how it relates to the Kasparov product. In Section 7 we prove Kucerovsky's theorem for half-closed chains.

Acknowledgements. We would like to thank Georges Skandalis for a highly stimulating remark concerning the "locality" of Kucerovsky's theorem.

This work also benefited from various conversations with Magnus Goffeng and Bram Mesland.

The authors gratefully acknowledge the Syddansk Universitet Odense and the Radboud University Nijmegen for their financial support in facilitating this collaboration.

During the initial stages of this research project the first author was supported by the Radboud excellence fellowship.

The first author was partially supported by the DFF-Research Project 2 "Automorphisms and Invariants of Operator Algebras", no. 701400145B and by the Villum Foundation (grant 7423).

The second author was partially supported by NWO under VIDIgrant 016.133.326.

\section{HALF-CLOSED CHAinS}

Let us fix two $\sigma$-unital $C^{*}$-algebras $A$ and $B$.

Let $E$ be a countably generated Hilbert $C^{*}$-module over $B$. We recall that a closed (densely defined) unbounded operator $D: \operatorname{Dom}(D) \rightarrow$ 
$E$ is said to be regular when it has a densely defined adjoint $D^{*}$ : $\operatorname{Dom}\left(D^{*}\right) \rightarrow E$ and when $1+D^{*} D: \operatorname{Dom}\left(D^{*} D\right) \rightarrow E$ has dense range. It follows from this definition that $1+D^{*} D: \operatorname{Dom}\left(D^{*} D\right) \rightarrow E$ is in fact densely defined and surjective, [LAN95, Lemma 9.1]. In particular we have a bounded adjointable inverse $\left(1+D^{*} D\right)^{-1}: E \rightarrow E$.

For two countably generated Hilbert $C^{*}$-modules $E$ and $F$ over $B$, we let $\mathbb{L}(E, F)$ and $\mathbb{K}(E, F)$ denote the bounded adjointable operators from $E$ to $F$ and the compact operators from $E$ to $F$, respectively. When $E=F$ we put $\mathbb{L}(E):=\mathbb{L}(E, F)$ and $\mathbb{K}(E):=\mathbb{K}(E, F)$. We let $\|\cdot\|_{\infty}: \mathbb{L}(E, F) \rightarrow[0, \infty)$ denote the operator norm.

The following definition is due to Hilsum, [HIL10, Section 3]:

Definition 1. A half-closed chain from $A$ to $B$ is a triple $(\mathscr{A}, E, D)$, where $\mathscr{A} \subseteq A$ is a norm-dense $*$-subalgebra, $E$ is a countably generated $C^{*}$-correspondence from $A$ to $B$ and $D: \operatorname{Dom}(D) \rightarrow E$ is a closed, symmetric and regular unbounded operator such that

(1) $a \cdot\left(1+D^{*} D\right)^{-1}$ is a compact operator on $E$ for all $a \in A$;

(2) $a\left(\operatorname{Dom}\left(D^{*}\right)\right) \subseteq \operatorname{Dom}(D)$ for all $a \in \mathscr{A}$;

(3) $[D, a]: \operatorname{Dom}(D) \rightarrow E$ extends to a bounded operator $d(a): E \rightarrow$ $E$ for all $a \in \mathscr{A}$.

$A$ half-closed chain $(\mathscr{A}, E, D)$ from $A$ to $B$ is said to be even when $E$ comes equipped with a $\mathbb{Z} / 2 \mathbb{Z}$-grading operator $\gamma: E \rightarrow E\left(\gamma=\gamma^{*}\right.$, $\left.\gamma^{2}=1\right)$, such that $[a, \gamma]=0$ for all $a \in A$ and $D \gamma=-\gamma D$.

$A$ half-closed chain which is not even is said to be odd.

Let $(\mathscr{A}, E, D)$ be a half-closed chain from $A$ to $B$. A few observations are in place:

(1) $d(a): E \rightarrow E, a \in \mathscr{A}$, is automatically adjointable with $d(a)^{*}=$ $-d\left(a^{*}\right)$.

(2) The difference

$$
D a-a D^{*}: \operatorname{Dom}\left(D^{*}\right) \rightarrow E \quad a \in \mathscr{A}
$$

extends to the bounded adjointable operator $d(a): E \rightarrow E$.

(3) $a \cdot\left(1+D D^{*}\right)^{-1} \in \mathbb{K}(E)$ for all $a \in A$. (Remark that $D^{*}$ is automatically regular by [LAN95, Proposition 9.5]).

We recall that a Kasparov module from $A$ to $B$ is a pair $(E, F)$ where $E$ is a countably generated $C^{*}$-correspondence from $A$ to $B$ and $F: E \rightarrow E$ is a bounded adjointable operator such that

$$
a \cdot\left(F-F^{*}\right), a \cdot\left(F^{2}-1\right),[F, a] \in \mathbb{K}(E),
$$

for all $a \in A$. A Kasparov module $(E, F)$ from $A$ to $B$ is even when it comes equipped with a $\mathbb{Z} / 2 \mathbb{Z}$-grading operator $\gamma: E \rightarrow E$ such that $[a, \gamma]=0$ for all $a \in A$ and $F \gamma+\gamma F=0$. Otherwise we say that $(E, F)$ is odd. 
For an unbounded regular operator $D: \operatorname{Dom}(D) \rightarrow E$ we let $F_{D}:=$ $D\left(1+D^{*} D\right)^{-1 / 2} \in \mathbb{L}(E)$ denote the bounded transform of $D$. We have that $F_{D}^{*}=F_{D^{*}}=D^{*}\left(1+D D^{*}\right)^{-1 / 2}$.

The next result creates the main link between half-closed chains and Kasparov modules. This result is due to Hilsum, [HIL10], and it generalizes the corresponding result of Baaj and Julg for unbounded Kasparov modules, BAJU83. Remark however that the condition $\left[F_{D}, a\right] \in \mathbb{K}(E), a \in A$, is for some reason left unproved in [HIL10, Theorem 3.2]. We therefore give a full proof of this commutator condition here:

Theorem 2. Suppose that $(\mathscr{A}, E, D)$ is a half-closed chain from $A$ to $B$. Then $\left(E, F_{D}\right)$ is a Kasparov module from $A$ to $B$ of the same parity as $(\mathscr{A}, E, D)$ and with the same $\mathbb{Z} / 2 \mathbb{Z}$-grading operator $\gamma: E \rightarrow E$ in the even case.

Proof. We have to show that $\left[F_{D}, a\right] \in \mathbb{K}(E)$ for all $a \in A$. Since the *-algebra $\mathscr{A} \subseteq A$ is dense in $C^{*}$-norm and since the $C^{*}$-algebra $\mathbb{K}(E) \subseteq$ $\mathbb{L}(E)$ is closed in operator norm it suffices to show that $\left[F_{D}, a\right] \cdot b \in \mathbb{K}(E)$ for all $a, b \in \mathscr{A}$.

We recall that

$$
\left(1+D^{*} D\right)^{-1 / 2}=\frac{1}{\pi} \int_{0}^{\infty} \lambda^{-1 / 2}\left(1+\lambda+D^{*} D\right)^{-1} d \lambda,
$$

where the integral converges absolutely in operator norm and where the integrand is continuous in operator norm. Remark here that $\|(1+$ $\left.\lambda+D^{*} D\right)^{-1} \|_{\infty} \leq(1+\lambda)^{-1}$ for all $\lambda \geq 0$.

For $a \in \mathscr{A}$ and $\lambda \geq 0$ we then compute that

$$
\begin{gathered}
{\left[D\left(1+\lambda+D^{*} D\right)^{-1}, a\right]} \\
=-D D^{*}\left(1+\lambda+D D^{*}\right)^{-1} d(a)\left(1+\lambda+D^{*} D\right)^{-1} \\
-D\left(1+\lambda+D^{*} D\right)^{-1} d(a) D\left(1+\lambda+D^{*} D\right)^{-1} \\
\quad+d(a)\left(1+\lambda+D^{*} D\right)^{-1}
\end{gathered}
$$

In particular, it holds for each $a, b \in \mathscr{A}$ that the map

$$
M:(0, \infty) \rightarrow \mathbb{L}(E) \quad M(\lambda):=\lambda^{-1 / 2}\left[D\left(1+\lambda+D^{*} D\right)^{-1}, a\right] b
$$

is continuous in operator norm and that $M(\lambda) \in \mathbb{K}(E)$ for all $\lambda \in$ $(0, \infty)$. Moreover, we have the estimate

$$
\|M(\lambda)\|_{\infty} \leq \lambda^{-1 / 2} \cdot\|d(a)\| \cdot 3 \cdot(1+\lambda)^{-1},
$$

for all $\lambda>0$. We may thus conclude that

$$
\left[F_{D}, a\right] b=\frac{1}{\pi} \int_{0}^{\infty} M(\lambda) d \lambda \in \mathbb{K}(E),
$$

for all $a, b \in \mathscr{A}$. This proves the theorem. 


\section{UNBOUNDED MODULAR CYCLES}

Let us fix $\sigma$-unital $C^{*}$-algebras $A$ and $B$ together with a dense *subalgebra $\mathscr{A} \subseteq A$.

The following definition is from [KAA15, Section 3]:

Definition 3. An unbounded modular cycle from $\mathscr{A}$ to $B$ is a triple $(E, D, \Delta)$ where $E$ is a countably generated $C^{*}$-correspondence from $A$ to $B, D: \operatorname{Dom}(D) \rightarrow E$ is an unbounded selfadjoint and regular operator, and $\Delta: E \rightarrow E$ is a bounded positive and selfadjoint operator with norm-dense image such that

(1) $a(i+D)^{-1}: E \rightarrow E$ is a compact operator for all $a \in A$;

(2) $(a+\lambda) \Delta$ has $\operatorname{Dom}(D) \subseteq E$ as an invariant submodule and

$$
D(a+\lambda) \Delta-\Delta(a+\lambda) D: \operatorname{Dom}(D) \rightarrow E
$$

extends to a bounded adjointable operator $d_{\Delta}(a, \lambda): E \rightarrow E$ for all $a \in \mathscr{A}, \lambda \in \mathbb{C}$.

(3) The supremum

$$
\sup _{\varepsilon>0}\left\|\left(\Delta^{1 / 2}+\varepsilon\right)^{-1} d_{\Delta}(a, \lambda)\left(\Delta^{1 / 2}+\varepsilon\right)^{-1}\right\|_{\infty}
$$

is finite for all $a \in \mathscr{A}, \lambda \in \mathbb{C}$.

(4) The sequence $\left\{\Delta(\Delta+1 / n)^{-1} a\right\}$ converges in operator norm to a for all $a \in A$.

An unbounded modular cycle is even when $E$ comes equipped with $a \mathbb{Z} / 2 \mathbb{Z}$-grading operator $\gamma: E \rightarrow E\left(\gamma=\gamma^{*}, \gamma^{2}=1\right)$, such that $[a, \gamma]=0$ for all $a \in A$ and $D \gamma=-\gamma D$.

An unbounded modular cycle is odd when it is not even.

Remark 4. Note that if $\Delta$ has a bounded inverse then (3) and (4) are automatic. If, in addition, $A$ is unital, $\Delta, \Delta^{-1} \in \mathscr{A}$ and $B=\mathbb{C}$ then the modular cycle $(E, D, \Delta)$ defines a twisted spectral triple in the sense of [CoMo08], with the twisting automorphism $\sigma: \mathscr{A} \rightarrow \mathscr{A}$ given by $\sigma(a)=\Delta a \Delta^{-1}$ for all $a \in \mathscr{A}$.

Remark 5. In [KAA15] it is assumed that $\mathscr{A}$ is equipped with a fixed operator space norm $\|\cdot\|_{1}: M_{n}(\mathscr{A}) \rightarrow[0, \infty), n \in \mathbb{N}$, such that the inclusion $\mathscr{A} \rightarrow A$ is completely bounded. In the above definition it is then required that the supremum in (3) is completely bounded in the sense that

$$
\sup _{\varepsilon>0}\left\|\left(\Delta^{1 / 2}+\varepsilon\right)^{-1} d_{\Delta}(a, 0)\left(\Delta^{1 / 2}+\varepsilon\right)^{-1}\right\|_{\infty} \leq C \cdot\|a\|_{1}
$$

for all $a \in M_{n}(\mathscr{A}), n \in \mathbb{N}$ (thus, the constant $C$ is independent of the size of the matrices). This structure is relevant for the construction of the unbounded Kasparov product, but will not play a role in the present text. 
As in the case of half-closed chains, each unbounded modular cycle represents an explicit class in KK-theory. This result can be found as KAA15, Theorem 9.1]. We state it here for the convenience of the reader. We recall that $F_{D}:=D\left(1+D^{2}\right)^{-1 / 2}$ denotes the bounded transform of $D: \operatorname{Dom}(D) \rightarrow E$ (but now $D$ is selfadjoint and regular).

Theorem 6. Suppose that $(E, D, \Delta)$ is an unbounded modular cycle from $\mathscr{A}$ to $B$. Then $\left(E, F_{D}\right)$ is a Kasparov module from $A$ to $B$ of the same parity as $(E, D, \Delta)$ and the same $\mathbb{Z} / 2 \mathbb{Z}$-grading operator $\gamma: E \rightarrow$ $E$ in the even case.

\section{LOCALIZATION OF REGULAR UNBOUNDED OPERATORS}

Let $E$ be a countably generated Hilbert $C^{*}$-module over a $\sigma$-unital $C^{*}$-algebra $B$ and let $D: \operatorname{Dom}(D) \rightarrow E$ be a closed, symmetric and regular unbounded operator.

Assumption 1. It will be assumed that $\Delta: E \rightarrow E$ is a bounded selfadjoint operator such that

(1) $\Delta\left(\operatorname{Dom}\left(D^{*}\right)\right) \subseteq \operatorname{Dom}(D)$;

(2) $D \Delta-\Delta D: \operatorname{Dom}(D) \rightarrow E$ extends to a bounded operator $d(\Delta)$ : $E \rightarrow E$.

Remark that it follows by the above assumption and the inclusion $D \subseteq D^{*}$ that

$$
D \Delta-\Delta D^{*}: \operatorname{Dom}\left(D^{*}\right) \rightarrow E
$$

also has $d(\Delta): E \rightarrow E$ as a bounded extension. Moreover, $d(\Delta): E \rightarrow$ $E$ is automatically adjointable with $d(\Delta)^{*}=-d(\Delta)$.

Before proving our first result, we notice that $D \Delta: \operatorname{Dom}(D \Delta) \rightarrow E$ is a closed unbounded operator on the domain

$$
\operatorname{Dom}(D \Delta):=\{\xi \in E \mid \Delta(\xi) \in \operatorname{Dom}(D)\} .
$$

A similar remark holds for $D^{*} \Delta: \operatorname{Dom}\left(D^{*} \Delta\right) \rightarrow E$.

Proposition 7. Suppose that the conditions in Assumption 1 hold. Then

$$
D \Delta=D^{*} \Delta
$$

and $D \Delta: \operatorname{Dom}(D \Delta) \rightarrow E$ is a regular unbounded operator with core $\operatorname{Dom}(D)$ and with

$$
(D \Delta)^{*}=D \Delta-d(\Delta) .
$$

In particular, we have that

$$
\operatorname{Dom}\left((D \Delta)^{*}\right)=\operatorname{Dom}(D \Delta) .
$$

Proof. We first claim that the unbounded operators $D \Delta: \operatorname{Dom}(D \Delta) \rightarrow$ $E$ and $D^{*} \Delta: \operatorname{Dom}\left(D^{*} \Delta\right) \rightarrow E$ are regular with cores $\operatorname{Dom}\left(D^{*}\right)$ and $\operatorname{Dom}(D)$, respectively, and with adjoints

$$
(D \Delta)^{*}=D \Delta-d(\Delta) \quad \text { and } \quad\left(D^{*} \Delta\right)^{*}=D^{*} \Delta-d(\Delta) .
$$


To prove this claim, we recall that $D: \operatorname{Dom}(D) \rightarrow E$ is regular by assumption, and we thus have that

$$
\left(\begin{array}{cc}
0 & D^{*} \\
D & 0
\end{array}\right): \operatorname{Dom}(D) \oplus \operatorname{Dom}\left(D^{*}\right) \rightarrow E \oplus E
$$

is selfadjoint and regular. Moreover, we have that

$$
\left(\begin{array}{cc}
0 & \Delta \\
\Delta & 0
\end{array}\right)\left(\operatorname{Dom}(D) \oplus \operatorname{Dom}\left(D^{*}\right)\right) \subseteq \operatorname{Dom}(D) \oplus \operatorname{Dom}\left(D^{*}\right)
$$

and the identities

$$
\begin{aligned}
{\left[\left(\begin{array}{cc}
0 & D^{*} \\
D & 0
\end{array}\right),\left(\begin{array}{cc}
0 & \Delta \\
\Delta & 0
\end{array}\right)\right] } & =\left(\begin{array}{cc}
D \Delta-\Delta D & 0 \\
0 & D \Delta-\Delta D^{*}
\end{array}\right) \\
& =\left(\begin{array}{cc}
d(\Delta) & 0 \\
0 & d(\Delta)
\end{array}\right)
\end{aligned}
$$

hold on $\operatorname{Dom}(D) \oplus \operatorname{Dom}\left(D^{*}\right)$. This means that $\left(\begin{array}{cc}0 & D^{*} \\ D & 0\end{array}\right)$ and $\left(\begin{array}{cc}0 & \Delta \\ \Delta & 0\end{array}\right)$ satisfy the conditions of [KAA17, Section 6] and we may conclude that

$$
\begin{aligned}
\left(\begin{array}{cc}
0 & D^{*} \\
D & 0
\end{array}\right)\left(\begin{array}{cc}
0 & \Delta \\
\Delta & 0
\end{array}\right)= & \left(\begin{array}{cc}
D^{*} \Delta & 0 \\
0 & D \Delta
\end{array}\right) \\
& : \operatorname{Dom}\left(D^{*} \Delta\right) \oplus \operatorname{Dom}(D \Delta) \rightarrow E \oplus E
\end{aligned}
$$

is a regular unbounded operator with

$$
\begin{aligned}
\left(\begin{array}{cc}
D^{*} \Delta & 0 \\
0 & D \Delta
\end{array}\right)^{*}= & \left(\begin{array}{cc}
D^{*} \Delta & 0 \\
0 & D \Delta
\end{array}\right)-\left(\begin{array}{cc}
d(\Delta) & 0 \\
0 & d(\Delta)
\end{array}\right) \\
& : \operatorname{Dom}\left(D^{*} \Delta\right) \oplus \operatorname{Dom}(D \Delta) \rightarrow E \oplus E
\end{aligned}
$$

Moreover, we know that $\left(\begin{array}{cc}D^{*} \Delta & 0 \\ 0 & D \Delta\end{array}\right): \operatorname{Dom}\left(D^{*} \Delta\right) \oplus \operatorname{Dom}(D \Delta) \rightarrow$ $E \oplus E$ has $\operatorname{Dom}(D) \oplus \operatorname{Dom}\left(D^{*}\right)$ as a core. This proves the claim.

To end the proof of the proposition, it now suffices to prove that $D \Delta=D^{*} \Delta$. To this end, we notice that

$$
\left(D^{*} \Delta\right)(\xi)=(D \Delta)(\xi) \quad \text { for all } \xi \in \operatorname{Dom}\left(D^{*}\right)
$$

Since $\operatorname{Dom}(D) \subseteq \operatorname{Dom}\left(D^{*}\right)$ is a core for $D^{*} \Delta$ we obtain from Equation (4.1) that $D^{*} \Delta \subseteq D \Delta$. Moreover, since $\operatorname{Dom}\left(D^{*}\right)$ is a core for $D \Delta$ we also obtain from Equation (4.1) that $D \Delta \subseteq D^{*} \Delta$. We conclude that $D \Delta=D^{*} \Delta$.

Assumption 2. It will be assumed that $x: E \rightarrow E$ is a bounded adjointable operator such that

(1) $x\left(\operatorname{Dom}\left(D^{*}\right)\right) \subseteq \operatorname{Dom}(D)$ and $x^{*}\left(\operatorname{Dom}\left(D^{*}\right)\right) \subseteq \operatorname{Dom}(D)$;

(2) $D x-x D$ and $D x^{*}-x^{*} D: \operatorname{Dom}(D) \rightarrow E$ extend to bounded operators $d(x)$ and $d\left(x^{*}\right): E \rightarrow E$, respectively. 
As above, $d(x)$ and $d\left(x^{*}\right): E \rightarrow E$ are automatically adjointable with $d(x)^{*}=-d\left(x^{*}\right)$. Moreover, $d(x)$ and $d\left(x^{*}\right)$ are bounded extensions of $D x-x D^{*}$ and $D x^{*}-x^{*} D^{*}: \operatorname{Dom}\left(D^{*}\right) \rightarrow E$, respectively.

We define the localization of $E$ (with respect to $x: E \rightarrow E$ ) as the Hilbert $C^{*}$-submodule $E_{x} \subseteq E$ given by the norm-closure of the image of $x$ :

$$
E_{x}:=\operatorname{cl}(\operatorname{Im}(x)) .
$$

We define $\Delta:=x x^{*}: E \rightarrow E$.

Lemma 8. Suppose that the conditions of Assumption 2 are satisfied. Then the unbounded operator

$$
D \Delta-d(x) x^{*}: \operatorname{Dom}(D \Delta) \rightarrow E
$$

is selfadjoint and regular and it has $\operatorname{Dom}(D) \subseteq \operatorname{Dom}(D \Delta)$ as a core. Moreover, we have that

$$
\left(D \Delta-d(x) x^{*}\right)(\xi)=\left(x D x^{*}\right)(\xi),
$$

for all $\xi \in \operatorname{Dom}\left(D x^{*}\right) \subseteq \operatorname{Dom}(D \Delta)$.

Proof. Clearly, $\Delta=x x^{*}: E \rightarrow E$ satisfied the conditions of Assumption 1 and it therefore follows from Proposition 7 that $D \Delta$ : $\operatorname{Dom}(D \Delta) \rightarrow E$ is regular with core $\operatorname{Dom}(D)$ and that

$$
(D \Delta)^{*}=D \Delta-d(\Delta)=D \Delta-d(x) x^{*}-x d\left(x^{*}\right) .
$$

Since $d(x) x^{*}: E \rightarrow E$ is a bounded adjointable operator, it follows by [Wor91, Section 2, Example 1] that $D \Delta-d(x) x^{*}: \operatorname{Dom}(D \Delta) \rightarrow E$ is regular. It is moreover clear that $\operatorname{Dom}(D)$ is also a core for $D \Delta-d(x) x^{*}$ and that

$$
\begin{aligned}
\left(D \Delta-d(x) x^{*}\right)^{*} & =(D \Delta)^{*}-\left(d(x) x^{*}\right)^{*} \\
& =D \Delta-d(x) x^{*}-x d\left(x^{*}\right)+x d\left(x^{*}\right)=D \Delta-d(x) x^{*},
\end{aligned}
$$

proving that our unbounded operator is selfadjoint as well. The final statement of the lemma is obvious.

Definition 9. Suppose that the conditions of Assumption 2 are satisfied. We define the localization of $D: \operatorname{Dom}(D) \rightarrow E$ (with respect to $x: E \rightarrow E$ ) as the closure of the unbounded symmetric operator

$$
x D x^{*}: \operatorname{Dom}(D) \cap E_{x} \rightarrow E_{x} .
$$

The localization of $D$ is denoted by

$$
D_{x}: \operatorname{Dom}\left(D_{x}\right) \rightarrow E_{x} .
$$

Remark that $x(\operatorname{Dom}(D)) \subseteq \operatorname{Dom}(D) \cap E_{x}$, implying that the localization $D_{x}$ is densely defined. 
Lemma 10. Suppose that the conditions of Assumption 2 are satisfied and let $r \in \mathbb{R}$ with $|r|>\left\|d\left(x^{*}\right) x\right\|_{\infty}$ be given. Then ir $+D x^{*} x$ : $\operatorname{Dom}\left(D x^{*} x\right) \rightarrow E$ is a bijection and the resolvent is a bounded adjointable operator $\left(\text { ir }+D x^{*} x\right)^{-1}: E \rightarrow E$ satisfying the relation

$$
\left(i r+D \Delta-d(x) x^{*}\right)^{-1} x=x\left(i r+D x^{*} x\right)^{-1} .
$$

Proof. By replacing $x$ with $x^{*}$ in Assumption 2 we see from Lemma 8 that the unbounded operator

$$
D x^{*} x-d\left(x^{*}\right) x: \operatorname{Dom}\left(D x^{*} x\right) \rightarrow E
$$

is selfadjoint and regular. In particular, we know that the resolvent $\left(i r+D x^{*} x-d\left(x^{*}\right) x\right)^{-1}: E \rightarrow E$ is a well-defined bounded adjointable operator. Since

$$
\left\|d\left(x^{*}\right) x\left(i r+D x^{*} x-d\left(x^{*}\right) x\right)^{-1}\right\|_{\infty} \leq\left\|d\left(x^{*}\right) x\right\|_{\infty} \cdot|r|^{-1}<1
$$

we may conclude that ir $+D x^{*} x: \operatorname{Dom}\left(D x^{*} x\right) \rightarrow E$ is a bijection and that the resolvent is a bounded adjointable operator. In fact, we have that

$$
\begin{aligned}
\left(i r+D x^{*} x\right)^{-1}= & \left(i r+D x^{*} x-d\left(x^{*}\right) x\right)^{-1} \\
& \cdot\left(1+d\left(x^{*}\right) x\left(i r+D x^{*} x-d\left(x^{*}\right) x\right)^{-1}\right)^{-1} .
\end{aligned}
$$

The relation in Equation Equation (4.2) now follows since

$$
\left(i r+D \Delta-d(x) x^{*}\right) x=\left(i r+x D x^{*}\right) x=x\left(i r+D x^{*} x\right)
$$

on $\operatorname{Dom}\left(D x^{*} x\right)$.

Proposition 11. Suppose that the conditions of Assumption 2 are satisfied. Then the localization of $D: \operatorname{Dom}(D) \rightarrow E$ with respect to $x: E \rightarrow E$ is a selfadjoint and regular unbounded operator

$$
D_{x}: \operatorname{Dom}\left(D_{x}\right) \rightarrow E_{x}
$$

with core $x(\operatorname{Dom}(D)) \subseteq \operatorname{Dom}\left(D_{x}\right)$. Moreover, we have the identity

$$
\left(i \mu+D_{x}\right)^{-1}(\xi)=\left(i \mu+D \Delta-d(x) x^{*}\right)^{-1}(\xi),
$$

for all $\xi \in E_{x}$ and all $\mu \in \mathbb{R} \backslash\{0\}$. In particular, $E_{x} \subseteq E$ is an invariant submodule for $\left(i \mu+D \Delta-d(x) x^{*}\right)^{-1}: E \rightarrow E$ for all $\mu \in \mathbb{R} \backslash\{0\}$.

Proof. To show that $D_{x}: \operatorname{Dom}\left(D_{x}\right) \rightarrow E_{x}$ is selfadjoint and regular, it suffices to verify that

$$
i r+x D x^{*}: x(\operatorname{Dom}(D)) \rightarrow E_{x}
$$

has dense image whenever $r \in \mathbb{R}$ satisfies $|r|>\left\|d\left(x^{*}\right) x\right\|_{\infty}$, see [LAN95, Lemma 9.7 and Lemma 9.8]. Let such an $r \in \mathbb{R}$ be given.

Clearly, $x^{*} x: E \rightarrow E$ satisfies the condition of Assumption 1 and it therefore follows from Proposition 7 that $D x^{*} x: \operatorname{Dom}\left(D x^{*} x\right) \rightarrow E$ is regular with core $\operatorname{Dom}(D) \subseteq E$. Combining this with Lemma 10 we may find a norm-dense submodule $\mathscr{E} \subseteq E$ such that

$$
\left(i r+D x^{*} x\right)^{-1}(\mathscr{E})=\operatorname{Dom}(D) .
$$


Moreover, we have that

$$
\left(i r+x D x^{*}\right) x\left(i r+D x^{*} x\right)^{-1}(\xi)=x(\xi) \quad \text { for all } \xi \in \mathscr{E} .
$$

Since $x(\mathscr{E}) \subseteq E_{x}$ is norm-dense and $x\left(i r+D x^{*} x\right)^{-1}(\mathscr{E})=x(\operatorname{Dom}(D))$, this proves the desired density result and hence that the localization $D_{x}: \operatorname{Dom}\left(D_{x}\right) \rightarrow E_{x}$ is selfadjoint and regular.

Let $\mu \in \mathbb{R} \backslash\{0\}$. The identity in Equation (4.3) can now be verified on the image of $i \mu+x D x^{*}: x(\operatorname{Dom}(D)) \rightarrow E_{x}$, but here it follows immediately since $\left(x D x^{*}\right)(\xi)=\left(D \Delta-d(x) x^{*}\right)(\xi)$ for all $\xi \in x(\operatorname{Dom}(D))$.

Remark 12. The result of Proposition 11 can be generalized by replacing the bounded adjointable operator $x: E \rightarrow E$ by a sequence of bounded adjointable operators $x_{n}: E \rightarrow E, n \in \mathbb{N}$, each of them satisfying the conditions of Assumption 2. Suppose moreover that the sums

$$
\sum_{n=1}^{\infty} x_{n} x_{n}^{*} \quad \text { and } \quad \sum_{n=1}^{\infty} d\left(x_{n}\right) d\left(x_{n}\right)^{*}
$$

are norm-convergent in $\mathbb{L}(E)$ (this can of course always be obtained by rescaling the operators $\left.x_{n}: E \rightarrow E, n \in \mathbb{N}\right)$.

In this context, we define the localization of $E$ with respect to the sequence $x=\left\{x_{n}\right\}$ as the closed submodule

$$
E_{x}:=\operatorname{cl}\left(\operatorname{span}_{\mathbb{C}}\left\{x_{n}(\xi) \mid n \in \mathbb{N}, \xi \in E\right\}\right) \subseteq E .
$$

The localization $D_{x}$ of $D: \operatorname{Dom}(D) \rightarrow E$ is defined as the closure of the symmetric unbounded operator

$$
\sum_{n=1}^{\infty} x_{n} D x_{n}^{*}: \operatorname{Dom}(D) \cap E_{x} \rightarrow E_{x} .
$$

As in Proposition 11, we then obtain that $D_{x}: \operatorname{Dom}\left(D_{x}\right) \rightarrow E_{x}$ is a selfadjoint and regular unbounded operator.

\section{LOCALIZATION OF HALF-CLOSED CHAINS}

Let $A$ and $B$ be $\sigma$-unital $C^{*}$-algebras. Throughout this section $(\mathscr{A}, E, D)$ will be a half-closed chain from $A$ to $B$. We denote by $\phi: A \rightarrow \mathbb{L}(E)$ the $*$-homomorphism that provides the left action of $A$ on $E$. Moreover, $x \in \mathscr{A}$ will be a fixed element.

Notice that $\phi(x): E \rightarrow E$ satisfies the condition of Assumption 2 with respect to the symmetric and regular unbounded operator $D$ : $\operatorname{Dom}(D) \rightarrow E$. Recall then that the localization of $E$ is the normclosed submodule $E_{x}:=\operatorname{cl}(\operatorname{Im}(\phi(x))) \subseteq E$ and that the localization $D_{x}$ of $D: \operatorname{Dom}(D) \rightarrow E$ is the closure of the symmetric unbounded operator

$$
\phi(x) D \phi\left(x^{*}\right): \operatorname{Dom}(D) \cap E_{x} \rightarrow E_{x} .
$$


By Proposition 11, the localization $D_{x}: \operatorname{Dom}\left(D_{x}\right) \rightarrow E_{x}$ is selfadjoint and regular. We put

$$
\Delta:=x x^{*} \in \mathscr{A} .
$$

By definition, the localization of $A$ with respect to $x \in A$ is the hereditary $C^{*}$-subalgebra of $A$ defined by

$$
A_{x}:=\operatorname{cl}\left(x A x^{*}\right) \subseteq A .
$$

The $*$-homomorphism $\phi: A \rightarrow \mathbb{L}(E)$ restricts to a $*$-homomorphism $\phi_{x}: A_{x} \rightarrow \mathbb{L}\left(E_{x}\right)$ and in this way $E_{x}$ becomes a $C^{*}$-correspondence from $A_{x}$ to $B$. We remark that $\Delta \in A_{x}$ and that $\phi_{x}(\Delta): E_{x} \rightarrow E_{x}$ is a bounded positive and selfadjoint operator with norm-dense image.

We define the $*$-subalgebra $\mathscr{A}_{x} \subseteq A_{x}$ as the intersection

$$
\mathscr{A}_{x}:=\mathscr{A} \cap A_{x} .
$$

Remark that $\mathscr{A}_{x} \subseteq A_{x}$ is automatically norm-dense.

When the half-closed chain $(\mathscr{A}, E, D)$ is even with $\mathbb{Z} / 2 \mathbb{Z}$-grading operator $\gamma: E \rightarrow E$, then $E_{x}$ can be equipped with the $\mathbb{Z} / 2 \mathbb{Z}$-grading operator $\left.\gamma\right|_{E_{x}}: E_{x} \rightarrow E_{x}$ obtained by restriction of $\gamma: E \rightarrow E$.

We are going to prove the following:

Theorem 13. Suppose that $(\mathscr{A}, E, D)$ is a half-closed chain and that $x$ is an element in $\mathscr{A}$. Then the triple $\left(E_{x}, D_{x}, \phi_{x}(\Delta)\right)$ is an unbounded modular cycle from $\mathscr{A}_{x}$ to $B$ of the same parity as $(\mathscr{A}, E, D)$ and with grading operator $\left.\gamma\right|_{E_{x}}: E_{x} \rightarrow E_{x}$ in the even case.

Proof. Clearly the $C^{*}$-correspondence $E_{x}$ is countably generated (since $E$ is countably generated by assumption). Moreover, we have already established that the unbounded operator $D_{x}: \operatorname{Dom}\left(D_{x}\right) \rightarrow E_{x}$ is selfadjoint and regular in Proposition 11 and that $\phi_{x}(\Delta): E_{x} \rightarrow E_{x}$ is bounded positive and selfadjoint with norm-dense image. So it only remains to check conditions (1), (2), (3) and (4) of Definition 3 . The last condition (4) follows immediately since $\Delta(\Delta+1 / n)^{-1} a \rightarrow a$ in $C^{*}$-norm for all $a \in A_{x}$. The remaining three conditions are proved in Proposition 15, Proposition 16 and Proposition 17 below.

We will refer to the unbounded modular cycle $\left(E_{x}, D_{x}, \phi_{x}(\Delta)\right)$ as the localization of the half-closed chain $(E, \phi, D)$ with respect to $x \in \mathscr{A}$.

We start by proving the compactness condition (1) of Definition 3 . We put

$$
\widetilde{D_{x}}:=D \phi(\Delta)-d(x) \phi\left(x^{*}\right): \operatorname{Dom}(D \phi(\Delta)) \rightarrow E
$$

and recall that $\widetilde{D_{x}}$ is a selfadjoint and regular unbounded operator by Lemma 8. We remark that $\widetilde{D_{x}}$ agrees with $D_{x}$ if and only if the image of $\phi(x): E \rightarrow E$ is norm-dense. In fact, when the image of $\phi(x)$ is not norm-dense then these two unbounded operators do not even act on the same Hilbert $C^{*}$-module. 
Lemma 14. We have the resolvent identity

$$
\begin{aligned}
& \left(\begin{array}{cc}
0 & \phi(\Delta) \\
\phi(\Delta) & 0
\end{array}\right)\left(\begin{array}{cc}
\left(i+\widetilde{D_{x}}\right)^{-1} & 0 \\
0 & \left(i+\widetilde{D_{x}}\right)^{-1}
\end{array}\right)-\left(\begin{array}{cc}
i & D^{*} \\
D & i
\end{array}\right)^{-1} \\
& \quad=\left(\begin{array}{cc}
i & D^{*} \\
D & i
\end{array}\right)^{-1}\left(\begin{array}{cc}
d(x) \phi\left(x^{*}\right)-i & i \phi(\Delta) \\
i \phi(\Delta) & d(x) \phi\left(x^{*}\right)-i
\end{array}\right)\left(i+\widetilde{D_{x}}\right)^{-1} .
\end{aligned}
$$

Proof. It suffices to notice that the identities

$$
\begin{gathered}
\left(\begin{array}{cc}
i & D^{*} \\
D & i
\end{array}\right)\left(\begin{array}{cc}
0 & \phi(\Delta) \\
\phi(\Delta) & 0
\end{array}\right)-\left(\begin{array}{cc}
i+\widetilde{D_{x}} & 0 \\
0 & i+\widetilde{D_{x}}
\end{array}\right) \\
\quad=\left(\begin{array}{cc}
D \phi(\Delta)-i-\widetilde{D_{x}} & i \phi(\Delta) \\
i \phi(\Delta) & D \phi(\Delta)-i-\widetilde{D_{x}}
\end{array}\right) \\
\quad=\left(\begin{array}{cc}
d(x) \phi\left(x^{*}\right)-i & i \phi(\Delta) \\
i \phi(\Delta) & d(x) \phi\left(x^{*}\right)-i
\end{array}\right)
\end{gathered}
$$

hold on $\operatorname{Dom}(D \phi(\Delta)) \oplus \operatorname{Dom}(D \phi(\Delta))$. Recall in this respect that $D \phi(\Delta)=D^{*} \phi(\Delta)$ by Proposition 7 .

Proposition 15. The bounded adjointable operator

$$
\phi_{x}(a)\left(i+D_{x}\right)^{-1}: E_{x} \rightarrow E_{x}
$$

is compact for all $a \in A_{x}$.

Proof. Notice that $\Delta \in A_{x}$ and that the left ideal $A_{x} \cdot \Delta \subseteq A_{x}$ is norm-dense. It thus suffices to show that $\phi_{x}(\Delta) \cdot\left(i+D_{x}\right)^{-1} \in \mathbb{K}\left(E_{x}\right)$.

We apply the notation $\mathbb{K}\left(E, E_{x}\right) \subseteq \mathbb{K}(E)$ for the closed right ideal generated by all compact operators on $E$ of the form $|\xi\rangle\langle\eta|$ with $\xi \in E_{x}$ and $\eta \in E$. Similarly, we let $\mathbb{K}\left(E_{x}, E\right) \subseteq \mathbb{K}(E)$ denote the closed left ideal generated by all compact operators of the form $|\eta\rangle\langle\xi|$ for $\xi \in E_{x}$ and $\eta \in E$. We remark that $\mathbb{K}\left(E_{x}, E\right)=\mathbb{K}\left(E, E_{x}\right)^{*}$.

Since $(E, \phi, D)$ is a half-closed chain we know that

$$
\left(\begin{array}{cc}
\phi(\Delta) & 0 \\
0 & \phi(\Delta)
\end{array}\right)\left(\begin{array}{cc}
i & D^{*} \\
D & i
\end{array}\right)^{-1} \in \mathbb{K}(E \oplus E)
$$

and it therefore follows from Lemma 14 that

$$
\phi(\Delta)^{2}\left(i+\widetilde{D_{x}}\right)^{-1} \in \mathbb{K}\left(E, E_{x}\right) .
$$

Since $(\phi(\Delta)+1 / n)^{-1} \phi(\Delta)^{2} \rightarrow \phi(\Delta)$ as $n \rightarrow \infty$ this implies that also $\phi(\Delta)\left(i+\widetilde{D_{x}}\right)^{-1} \in \mathbb{K}\left(E, E_{x}\right)$ and thus that $\left(-i+\widetilde{D_{x}}\right)^{-1} \phi(\Delta) \in \mathbb{K}\left(E_{x}, E\right)$. We may thus conclude that $\phi(\Delta)\left(1+{\widetilde{D_{x}}}^{2}\right)^{-1} \phi(\Delta) \in \mathbb{K}\left(E, E_{x}\right) \cdot \mathbb{K}\left(E_{x}, E\right)$ restricts to a compact operator on the Hilbert $C^{*}$-module $E_{x} \subseteq E$. But this proves the present proposition since we have from Proposition 11 that

$$
\phi_{x}(\Delta)\left(1+D_{x}^{2}\right)^{-1} \phi_{x}(\Delta)=\left.\left(\phi(\Delta)\left(1+{\widetilde{D_{x}}}^{2}\right)^{-1} \phi(\Delta)\right)\right|_{E_{x}} .
$$


We continue by proving the twisted commutator condition (2) of Definition 3 .

Proposition 16. Let $a \in \mathscr{A}_{x}, \lambda \in \mathbb{C}$. Then $\left(\phi_{x}(a)+\lambda\right) \phi_{x}(\Delta): E_{x} \rightarrow$ $E_{x}$ has $\operatorname{Dom}\left(D_{x}\right) \subseteq E_{x}$ as an invariant submodule and

$$
D_{x}\left(\phi_{x}(a)+\lambda\right) \phi_{x}(\Delta)-\phi_{x}(\Delta)\left(\phi_{x}(a)+\lambda\right) D_{x}: \operatorname{Dom}\left(D_{x}\right) \rightarrow E_{x}
$$

extends to a bounded adjointable operator $d_{\Delta}(a, \lambda): E_{x} \rightarrow E_{x}$. In fact we have that

$$
d_{\Delta}(a, \lambda)=\left.\left(\phi(x) d\left(x^{*}(a+\lambda) x\right) \phi\left(x^{*}\right)\right)\right|_{E_{x}} .
$$

Proof. Let $\xi \in \operatorname{Dom}(D) \cap E_{x}$. We then have that

$$
\left(\phi_{x}(a)+\lambda\right) \phi_{x}(\Delta)(\xi) \in \operatorname{Dom}(D) \cap E_{x}
$$

and that

$$
\begin{gathered}
D_{x}\left(\phi_{x}(a)+\lambda\right) \phi_{x}(\Delta)(\xi)-\phi_{x}(\Delta)\left(\phi_{x}(a)+\lambda\right) D_{x}(\xi) \\
=\phi(x) D \phi\left(x^{*}\right)(\phi(a)+\lambda) \phi\left(x x^{*}\right)(\xi) \\
-\phi\left(x x^{*}\right)(\phi(a)+\lambda) \phi(x) D \phi\left(x^{*}\right)(\xi) \\
=\phi(x) d\left(x^{*}(a+\lambda) x\right) \phi\left(x^{*}\right)(\xi) .
\end{gathered}
$$

Since $\operatorname{Dom}(D) \cap E_{x}$ is a core for the localization $D_{x}: \operatorname{Dom}\left(D_{x}\right) \rightarrow E_{x}$, this proves the proposition.

We finally prove the supremum condition (3) of Definition 3 .

Proposition 17. Let $a \in \mathscr{A}_{x}, \lambda \in \mathbb{C}$. Then we have that

$$
\sup _{\varepsilon>0}\left\|\left(\phi_{x}(\Delta)^{1 / 2}+\varepsilon\right)^{-1} d_{\Delta}(a, \lambda)\left(\phi_{x}(\Delta)^{1 / 2}+\varepsilon\right)^{-1}\right\|_{\infty}<\infty .
$$

Proof. This follows immediately from Proposition 16. Indeed, the operator norm of

$$
\left(\phi_{x}(\Delta)^{1 / 2}+\varepsilon\right)^{-1} \phi(x): E \rightarrow E_{x}
$$

is bounded by 1 for all $\varepsilon>0$.

Remark 18. One may equip $\mathscr{A}_{x}$ with the operator space norm $\|\cdot\|_{1}$ : $M_{n}\left(\mathscr{A}_{x}\right) \rightarrow[0, \infty), n \in \mathbb{N}$, defined by

$$
\|a\|_{1}:=\sup \left\{\|a\|,\|d(a)\|_{\infty}\right\} \quad \text { for all } a \in M_{n}\left(\mathscr{A}_{x}\right),
$$

where the norms inside the supremum are the $C^{*}$-norm on $M_{n}(A)$ and the operator-norm on $\mathbb{L}\left(E^{\oplus n}\right)$, respectively. Clearly, the inclusion $\mathscr{A}_{x} \rightarrow A_{x}$ is then completely bounded. It is moreover possible to find a constant $C>0$ such that

$$
\sup _{\varepsilon>0}\left\|\left(\phi_{x}(\Delta)^{1 / 2}+\varepsilon\right)^{-1} d_{\Delta}(a, 0)\left(\phi_{x}(\Delta)^{1 / 2}+\varepsilon\right)^{-1}\right\|_{\infty} \leq C \cdot\|a\|_{1},
$$

for all $a \in M_{n}\left(\mathscr{A}_{x}\right)$. Cf. Remark 5 . 


\section{Localization as an unbounded Kasparov product}

In this section we continue under the conditions spelled out in the beginning of Section 5. We thus have a half-closed chain $(\mathscr{A}, E, D)$ and an element $x \in \mathscr{A}$.

The element $x \in \mathscr{A}$ provides us with a closed right ideal $I_{x} \subseteq A$ defined as the norm-closure:

$$
I_{x}:=\operatorname{cl}(x A) .
$$

In particular, we may consider $I_{x}$ as a countably generated Hilbert $C^{*}$ module over $A$. The hereditary $C^{*}$-subalgebra $A_{x}=\operatorname{cl}\left(x A x^{*}\right) \subseteq A$ can be identified with the compact operators on $I_{x}$ via the $*$-homomorphism $\psi: A_{x} \rightarrow \mathbb{L}\left(I_{x}\right)$ induced by the multiplication in $A$. We thus obtain an even Kasparov module $\left(I_{x}, 0\right)$ from $A_{x}$ to $A$ with corresponding class $\left[I_{x}, 0\right] \in K K_{0}\left(A_{x}, A\right)$ in KK-theory.

Moreover, by Theorem 2, our half-closed chain $(\mathscr{A}, E, D)$ (of parity $p \in\{0,1\})$ yields a Kasparov module $\left(E, F_{D}\right)$ from $A$ to $B$ with corresponding class $\left[E, F_{D}\right] \in K K_{p}(A, B)$.

Finally, the unbounded modular cycle $\left(\mathscr{A} \cap A_{x}, E_{x}, \phi_{x}(\Delta)\right)$ constructed in Section 5 yields a Kasparov module $\left(E_{x}, F_{D_{x}}\right)$ from $A_{x}$ to $B$ with corresponding class $\left[E_{x}, F_{D_{x}}\right] \in K K_{p}\left(A_{x}, B\right)$, see Theorem 6 .

In this section we will prove the following theorem:

Theorem 19. Suppose that $(\mathscr{A}, E, D)$ is a half-closed chain, that $x \in$ $\mathscr{A}$ and that $A_{x}$ is separable. Then we have the identity

$$
\left[E_{x}, F_{D_{x}}\right]=\left[I_{x}, 0\right] \widehat{\otimes}_{A}\left[E, F_{D}\right]
$$

in $K K_{p}\left(A_{x}, B\right)$, where $\widehat{\otimes}_{A}: K K_{0}\left(A_{x}, A\right) \times K K_{p}(A, B) \rightarrow K K_{p}\left(A_{x}, B\right)$ denotes the Kasparov product.

Proof. The $C^{*}$-correspondence $E_{x}$ from $A_{x}$ to $A$ is unitarily isomorphic to the interior tensor product of $C^{*}$-correspondences $I_{x} \widehat{\otimes}_{\phi} E$ (via the unitary isomorphism $x a \widehat{\otimes} \xi \mapsto \phi(x a)(\xi))$. For each $a \in A$, we define the bounded adjointable operator $T_{x a}: E \rightarrow E_{x}$ by $\xi \mapsto \phi(x a)(\xi)$. By COSK84, Theorem A.3] it suffices to prove the connection condition, thus that

$$
\begin{aligned}
& F_{D_{x}} T_{x a}-T_{x a} F_{D}, \\
& F_{D_{x}} T_{x a}-T_{x a} F_{D^{*}} \in \mathbb{K}\left(E, E_{x}\right)
\end{aligned}
$$

for all $a \in A$. Indeed, the positivity condition of CoSk84, Theorem A.3] is obviously satisfied since the bounded adjointable operator in the Kasparov module $\left(I_{x}, 0\right)$ from $A_{x}$ to $A$ is trivial. See also Section 7 for more details.

However, since $T_{x a}=T_{x} \phi(a): E \rightarrow E_{x}$ and $\phi(a)\left(F_{D}-F_{D^{*}}\right) \in \mathbb{K}(E)$ it suffices to prove the first of these inclusions. This proof will occupy the remainder of this section, see Proposition 26. 
Remark 20. In the case where $x A \subseteq A$ is norm-dense and $A$ is separable, we have that $\left(I_{x}, 0\right)=(A, 0)$ and it therefore follows from the above theorem that the two Kasparov modules $\left(E_{x}, F_{D_{x}}\right)$ and $\left(E, F_{D}\right)$ represents the same class in $K K_{p}(A, B)$.

6.1. The modular transform. We continue working under the general assumptions stated in the beginning of Section 5. We recall that $\Delta:=x x^{*}$. We will in the following suppress the $*$-homomorphism $\phi_{x}: A_{x} \rightarrow \mathbb{L}\left(E_{x}\right)$.

For each $\lambda \geq 0$, we introduce the notation

$$
\begin{aligned}
& R_{x}\left(\lambda \Delta^{2}\right):=\left(1+\lambda \Delta^{2}+D_{x}^{2}\right)^{-1} \in \mathbb{L}\left(E_{x}\right) \\
& R_{x}(\lambda):=\left(1+\lambda+D_{x}^{2}\right)^{-1} \in \mathbb{L}\left(E_{x}\right) .
\end{aligned}
$$

In general, we are not able to estimate the norm of $R_{x}\left(\lambda \Delta^{2}\right)$ from above by $(1+\lambda)^{-1}$ since $\Delta: E_{x} \rightarrow E_{x}$ may have zero in the spectrum. Instead, we recall the following basic estimate from [KAA15, Section 11]:

$$
\left\|\Delta R_{x}\left(\lambda \Delta^{2}\right) \Delta\right\|_{\infty} \leq \frac{2}{(1+\lambda)} \quad \forall \lambda \geq 0
$$

The next definition is from [KAA15, Section 8]:

Definition 21. The modular transform of the unbounded modular cycle $\left(E_{x}, D_{x}, \Delta\right)$ is the unbounded operator

$$
G_{\left(D_{x}, \Delta\right)}: \Delta\left(\operatorname{Dom}\left(D_{x}\right)\right) \rightarrow E_{x}
$$

defined by

$$
G_{\left(D_{x}, \Delta\right)}: \eta \mapsto \frac{1}{\pi} \int_{0}^{\infty} \lambda^{-1 / 2} \Delta\left(1+\lambda \Delta^{2}+D_{x}^{2}\right)^{-1} D_{x}(\eta) d \lambda
$$

We remark that $G_{\left(D_{x}, \Delta\right)}: \Delta\left(\operatorname{Dom}\left(D_{x}\right)\right) \rightarrow E_{x}$ is well-defined. Indeed, for $\eta=\Delta(\xi)$ with $\xi \in \operatorname{Dom}\left(D_{x}\right)$ we have from Proposition 16 that

$$
\begin{aligned}
& \Delta R_{x}\left(\lambda \Delta^{2}\right) D_{x}(\eta) \\
& \quad=\Delta R_{x}\left(\lambda \Delta^{2}\right) \Delta D_{x}(\xi)+\Delta R_{x}\left(\lambda \Delta^{2}\right) x d\left(x^{*} x\right) x^{*}(\xi) .
\end{aligned}
$$

Using the estimate from Equation (6.2), we may thus find a constant $C>0$ such that

$$
\left\|\Delta\left(1+\lambda \Delta^{2}+D_{x}^{2}\right)^{-1} D_{x}(\eta)\right\| \leq C \cdot(1+\lambda)^{-3 / 4} \quad \forall \lambda \geq 0,
$$

implying that the integral in Equation (6.3) converges absolutely in the norm on $E_{x}$.

The following result is a consequence of [KAA15, Theorem 8.1]:

Theorem 22. The difference

$$
F_{D_{x}} \Delta^{6}-G_{\left(D_{x}, \Delta\right)} \Delta^{6}: \operatorname{Dom}\left(D_{x}\right) \rightarrow E_{x}
$$

extends to a compact operator on $E_{x}$. 
Notice that the above result implies that the unbounded operator

$$
G_{\left(D_{x}, \Delta\right)} \Delta^{6}: \operatorname{Dom}\left(D_{x}\right) \rightarrow E_{x}
$$

extends to a bounded adjointable operator on $E_{x}$.

6.2. The connection condition. We will continue working under the assumptions of Section 5 .

We recall from Lemma 8 that

$$
\widetilde{D_{x}}:=D \phi(\Delta)-d(x) \phi\left(x^{*}\right): \operatorname{Dom}(D \phi(\Delta)) \rightarrow E
$$

is a selfadjoint and regular unbounded operator and we put

$$
\begin{aligned}
& \widetilde{R_{x}}\left(\lambda \phi\left(\Delta^{2}\right)\right):=\left(1+\lambda \phi\left(\Delta^{2}\right)+\left(\widetilde{D_{x}}\right)^{2}\right)^{-1} \in \mathbb{L}(E) \\
& R(\lambda):=\left(1+\lambda+D^{*} D\right)^{-1} \in \mathbb{L}(E),
\end{aligned}
$$

for all $\lambda \geq 0$.

Lemma 23. For each $\lambda \geq 0$, we have the identity

$$
\begin{aligned}
R(\lambda)- & \widetilde{R_{x}}\left(\lambda \phi\left(\Delta^{2}\right)\right) \phi\left(\Delta^{2}\right) \\
= & \widetilde{R_{x}}\left(\lambda \phi\left(\Delta^{2}\right)\right)\left(1-\phi\left(\Delta^{2}\right)+\phi(x) d\left(x^{*} x x^{*}\right) D\right) R(\lambda) \\
& \quad+\left(\widetilde{D_{x}} \widetilde{R_{x}}\left(\lambda \phi\left(\Delta^{2}\right)\right)\right)^{*} \phi(x) d\left(x^{*}\right) R(\lambda)
\end{aligned}
$$

of bounded adjointable operators on $E$.

Proof. We have the identities

$$
\begin{aligned}
& 1-\widetilde{R_{x}}\left(\lambda \phi\left(\Delta^{2}\right)\right) \phi\left(\Delta^{2}\right)\left(1+\lambda+D^{*} D\right) \\
&=1-\widetilde{R_{x}}\left(\lambda \phi\left(\Delta^{2}\right)\right)\left(1+\lambda \phi\left(\Delta^{2}\right)+\phi(x) D \phi\left(x^{*} x x^{*}\right) D\right) \\
& \quad+\widetilde{R_{x}}\left(\lambda \phi\left(\Delta^{2}\right)\right)\left(1-\phi\left(\Delta^{2}\right)+\phi(x) d\left(x^{*} x x^{*}\right) D\right) \\
&=\left(\widetilde{D_{x}} \widetilde{R_{x}}\left(\lambda \phi\left(\Delta^{2}\right)\right)\right)^{*} \phi(x) d\left(x^{*}\right) \\
& \quad+\widetilde{R_{x}}\left(\lambda \phi\left(\Delta^{2}\right)\right)\left(1-\phi\left(\Delta^{2}\right)+\phi(x) d\left(x^{*} x x^{*}\right) D\right)
\end{aligned}
$$

on $\operatorname{Dom}\left(D^{*} D\right)$. But this proves the lemma after multiplying with $R(\lambda)=\left(1+\lambda+D^{*} D\right)^{-1}$ from the right.

For each $y \in I_{x}=\operatorname{cl}(x A)$, we recall that $T_{y}: E \rightarrow E_{x}$ denotes the bounded adjointable operator $T_{y}: \xi \mapsto \phi(y)(\xi)$. Notice then that it follows from Proposition 11 that

$$
T_{\Delta} \widetilde{R_{x}}\left(\lambda \phi\left(\Delta^{2}\right)\right) \phi(\Delta)=\Delta R_{x}\left(\lambda \Delta^{2}\right) T_{\Delta}: E \rightarrow E_{x} .
$$

Lemma 24. The difference

$$
T_{\Delta} R(\lambda) D \phi(\Delta)-\Delta R_{x}\left(\lambda \Delta^{2}\right) D_{x} T_{\Delta^{2}}: \operatorname{Dom}(D \phi(\Delta)) \rightarrow E_{x}
$$

extends to a compact operator $M_{\lambda}: E \rightarrow E_{x}$ for all $\lambda \geq 0$. Moreover, there exists a constant $C>0$ such that

$$
\left\|M_{\lambda}\right\|_{\infty} \leq C \cdot(1+\lambda)^{-3 / 4} \quad \forall \lambda \geq 0 .
$$


Proof. Since $(\mathscr{A}, E, D)$ is a half-closed chain and $\left(E_{x}, D_{x}, \Delta\right)$ is an unbounded modular cycle we obtain that the difference

$$
T_{\Delta} R(\lambda) D \phi(\Delta)-\Delta R_{x}\left(\lambda \Delta^{2}\right) D_{x} T_{\Delta^{2}}: \operatorname{Dom}(D \phi(\Delta)) \rightarrow E_{x}
$$

extends to a compact operator $M_{\lambda}: E \rightarrow E_{x}$ for all $\lambda \geq 0$. Indeed, this is already true for each of the terms viewed separately. So we only need to prove the norm-estimate. To this end, we let $\xi \in \operatorname{Dom}(D \phi(\Delta))$ and compute that

$$
\begin{gathered}
\left(T_{\Delta} R(\lambda) D \phi(\Delta)-\Delta R_{x}\left(\lambda \Delta^{2}\right) D_{x} T_{\Delta^{2}}\right)(\xi) \\
=T_{\Delta} R(\lambda) D \phi(\Delta)(\xi)-T_{\Delta} \widetilde{R}_{x}\left(\lambda \phi\left(\Delta^{2}\right)\right) \phi(x) D \phi\left(x^{*} \Delta^{2}\right)(\xi) \\
=T_{\Delta} R(\lambda) D \phi(\Delta)(\xi)-T_{\Delta} \widetilde{R}_{x}\left(\lambda \phi\left(\Delta^{2}\right)\right) \phi\left(\Delta^{2}\right) D \phi(\Delta)(\xi) \\
\quad-T_{\Delta} \widetilde{R_{x}}\left(\lambda \phi\left(\Delta^{2}\right)\right) \phi(x) d\left(x^{*} x x^{*}\right) \phi(\Delta)(\xi) .
\end{gathered}
$$

Since $\left\|T_{\Delta} \widetilde{R_{x}}\left(\lambda \phi\left(\Delta^{2}\right)\right) \phi(x)\right\|_{\infty} \leq 2^{3 / 4} \cdot(1+\lambda)^{-3 / 4}$ by the estimate in Equation 6.2) we may focus on the difference

$$
T_{\Delta} R(\lambda) D \phi(\Delta)(\xi)-T_{\Delta} \widetilde{R_{x}}\left(\lambda \phi\left(\Delta^{2}\right)\right) \phi\left(\Delta^{2}\right) D \phi(\Delta)(\xi) .
$$

However, using Lemma 23 we get that

$$
\begin{aligned}
T_{\Delta} R(\lambda) D \phi(\Delta)(\xi)-T_{\Delta} \widetilde{R_{x}}\left(\lambda \phi\left(\Delta^{2}\right)\right) \phi\left(\Delta^{2}\right) D \phi(\Delta)(\xi) \\
=T_{\Delta} \widetilde{R_{x}}\left(\lambda \phi\left(\Delta^{2}\right)\right)\left(1-\phi\left(\Delta^{2}\right)+\phi(x) d\left(x^{*} x x^{*}\right) D\right) R(\lambda) D \phi(\Delta)(\xi) \\
\quad+T_{\Delta}\left(\widetilde{D_{x}} \widetilde{R_{x}}\left(\lambda \phi\left(\Delta^{2}\right)\right)\right)^{*} \phi(x) d\left(x^{*}\right) R(\lambda) D \phi(\Delta)(\xi) .
\end{aligned}
$$

The result of the lemma then follows from the basic estimate $\|D R(\lambda)\|_{\infty} \leq$ $(1+\lambda)^{-1 / 2}$ and the estimate in Equation $(6.2$ a few times.

Proposition 25. The difference

$$
T_{\Delta^{2}} F_{D}-G_{\left(D_{x}, \Delta\right)} T_{\Delta^{2}}: \operatorname{Dom}(D) \rightarrow E_{x}
$$

extends to a compact operator from $E$ to $E_{x}$.

Proof. Since $\phi(\Delta) F_{D}-F_{D^{*}} \phi(\Delta): E \rightarrow E$ is compact, we only need to show that

$$
T_{\Delta} F_{D^{*}} \phi(\Delta)-G_{\left(D_{x}, \Delta\right)} T_{\Delta^{2}}: \operatorname{Dom}(D) \rightarrow E_{x}
$$

extends to a compact operator from $E$ to $E_{x}$. Now, recall that

$$
T_{\Delta} F_{D^{*}} \phi(\Delta)(\xi)=\frac{1}{\pi} \int_{0}^{\infty} \lambda^{-1 / 2} T_{\Delta}\left(1+\lambda+D^{*} D\right)^{-1} D \phi(\Delta)(\xi) d \lambda
$$


for all $\xi \in \operatorname{Dom}(D)$. The result of the proposition now follows by Lemma 24 since

$$
\begin{aligned}
& T_{\Delta} D^{*}\left(1+D D^{*}\right)^{-1 / 2} \phi(\Delta)(\xi)-G_{\left(D_{x}, \Delta\right)} T_{\Delta^{2}}(\xi) \\
&= \frac{1}{\pi} \int_{0}^{\infty} \lambda^{-1 / 2}\left(T_{\Delta}\left(1+\lambda+D^{*} D\right)^{-1} D \phi(\Delta)\right. \\
&\left.\quad-\Delta R_{x}\left(\lambda \Delta^{2}\right) D_{x} T_{\Delta^{2}}\right)(\xi) d \lambda \\
&=\frac{1}{\pi} \int_{0}^{\infty} \lambda^{-1 / 2} M_{\lambda}(\xi) d \lambda .
\end{aligned}
$$

Remark that it follows from the above proposition that the unbounded operator

$$
G_{\left(D_{x}, \Delta\right)} T_{\Delta^{2}}: \operatorname{Dom}(D) \rightarrow E_{x}
$$

extends to a bounded adjointable operator on $E_{x}$.

Proposition 26. The difference

$$
F_{D_{x}} T_{x a}-T_{x a} F_{D}: E \rightarrow E_{x}
$$

is a compact operator for all $a \in A$.

Proof. Since $\left[\phi(b), F_{D}\right] \in \mathbb{K}(E)$ for all $b \in A$ and since $\Delta^{7}(1 / n+$ $\left.\Delta^{7}\right)^{-1} x \rightarrow x$ in the norm on $A$, it suffices to show that

$$
F_{D_{x}} T_{\Delta^{7}}-T_{\Delta^{7}} F_{D}: E \rightarrow E_{x}
$$

is a compact operator. But now Proposition 25 and Theorem 22 imply that the following identities hold modulo $\mathbb{K}\left(E, E_{x}\right)$ :

$$
\begin{aligned}
F_{D_{x}} T_{\Delta^{7}}-T_{\Delta^{7}} F_{D} & \sim F_{D_{x}} T_{\Delta^{7}}-T_{\Delta^{2}} F_{D} \phi\left(\Delta^{5}\right) \\
& \sim F_{D_{x}} T_{\Delta^{7}}-\operatorname{cl}\left(G_{\left(D_{x}, \Delta\right)} T_{\Delta^{2}}\right) \phi\left(\Delta^{5}\right) \\
& =F_{D_{x}} \Delta^{6} T_{\Delta}-\operatorname{cl}\left(G_{\left(D_{x}, \Delta\right)} \Delta^{6}\right) T_{\Delta} \sim 0 .
\end{aligned}
$$

\section{KuCEROVSKY'S THEOREM}

Let us fix three $C^{*}$-algebras $A, B$ and $C$ with $A$ separable and $B$ and $C$ both $\sigma$-unital. Throughout this section we will assume that $\left(\mathscr{A}, E_{1}, D_{1}\right),\left(\mathscr{B}, E_{2}, D_{2}\right)$ and $(\mathscr{A}, E, D)$ are even half-closed chains from $A$ to $B$, from $B$ to $C$ and from $A$ to $C$, respectively. We denote the associated $*$-homomorphisms by $\phi_{1}: A \rightarrow \mathbb{L}\left(E_{1}\right), \phi_{2}: B \rightarrow \mathbb{L}\left(E_{2}\right)$ and $\phi: A \rightarrow \mathbb{L}(E)$ and the $\mathbb{Z} / 2 \mathbb{Z}$-grading operators by $\gamma_{1}: E_{1} \rightarrow E_{1}$, $\gamma_{2}: E_{2} \rightarrow E_{2}$ and $\gamma: E \rightarrow E$, respectively. We will moreover assume that $E:=E_{1} \widehat{\otimes}_{\phi_{2}} E_{2}$ agrees with the interior tensor product of the $C^{*}$-correspondences $E_{1}$ and $E_{2}$. In particular, we assume that $\phi(a)=\phi_{1}(a) \widehat{\otimes} 1$ for all $a \in A$ and that $\gamma=\gamma_{1} \widehat{\otimes} \gamma_{2}$.

We will denote the bounded transforms of our half-closed chains by $\left(E_{1}, F_{D_{1}}\right),\left(E_{2}, F_{D_{2}}\right)$ and $\left(E, F_{D}\right)$ and the corresponding classes in KK-theory by $\left[E_{1}, F_{D_{1}}\right] \in K K_{0}(A, B),\left[E_{2}, F_{D_{2}}\right] \in K K_{0}(B, C)$ and 
$\left[E, F_{D}\right] \in K K_{0}(A, C)$. We may then form the interior Kasparov product

$$
\left[E_{1}, F_{D_{1}}\right] \widehat{\otimes}_{B}\left[E_{2}, F_{D_{2}}\right] \in K K_{0}(A, C)
$$

and it becomes a highly relevant question to find an explicit formula for this class in $K K_{0}(A, C)$.

In this section we shall find conditions on the half-closed chains $\left(\mathscr{A}, E_{1}, D_{1}\right),\left(\mathscr{B}, E_{2}, D_{2}\right)$ and $(\mathscr{A}, E, D)$ entailing that the identity

$$
\left[E, F_{D}\right]=\left[E_{1}, F_{D_{1}}\right] \widehat{\otimes}_{B}\left[E_{2}, F_{D_{2}}\right]
$$

holds in $K K_{0}(A, C)$. This kind of theorem was first proved by Kucerovsky in [KUC97] under the stronger assumption that the half-closed chains $\left(\mathscr{A}, E_{1}, D_{1}\right),\left(\mathscr{B}, E_{2}, D_{2}\right)$ and $(\mathscr{A}, E, D)$ were in fact unbounded Kasparov modules. Thus under the strong assumption that all the involved symmetric and regular unbounded operators were in fact selfadjoint. As in the case of Kucerovsky's theorem we rely on the work of Connes and Skandalis for computing the interior Kasparov product, see CoSk84.

We recall from [CoSk84, Theorem A.3] that an even Kasparov module $(E, F)$ from $A$ to $C$ is the Kasparov product of the even Kasparov modules $\left(E_{1}, F_{1}\right)$ and $\left(E_{2}, F_{2}\right)$ from $A$ to $B$ and from $B$ to $C$, respectively, when the following holds:

- $E=E_{1} \widehat{\otimes}_{\phi_{2}} E_{2}, \phi=\phi_{1} \widehat{\otimes} 1$.

- For every homogeneous $\xi \in E_{1}$ we have that

$$
F T_{\xi}-(-1)^{\partial \xi} T_{\xi} F_{2}, F^{*} T_{\xi}-(-1)^{\partial \xi} T_{\xi} F_{2}^{*} \in \mathbb{K}\left(E_{2}, E\right),
$$

where $T_{\xi}: E_{2} \rightarrow E$ is defined by $T_{\xi}(y):=\xi \widehat{\otimes} \eta$ for all $\eta \in E_{2}$ and where $\partial \xi \in\{0,1\}$ denotes the degree of $\xi \in E_{1}$.

- There exists a $\nu<2$ such that

$$
\left(\left(F_{1} \widehat{\otimes} 1\right)^{*} \cdot F^{*}+F \cdot\left(F_{1} \widehat{\otimes} 1\right)\right) \cdot \phi\left(a^{*} a\right)+\nu \cdot \phi\left(a^{*} a\right)
$$

is positive in the Calkin algebra $\mathbb{L}(E) / \mathbb{K}(E)$ for all $a \in A$.

The condition in Equation $(7.1)$ is often referred to as the connection condition and the condition in Equation $(7.2)$ is referred to as the positivity condition.

Before we state our conditions on half-closed chains we recall that the odd symmetric and regular unbounded operator $D_{1}: \operatorname{Dom}\left(D_{1}\right) \rightarrow E_{1}$ can be promoted to an odd symmetric and regular unbounded operator $D_{1} \widehat{\otimes} 1: \operatorname{Dom}\left(D_{1} \widehat{\otimes} 1\right) \rightarrow E_{1} \widehat{\otimes}_{\phi_{2}} E_{2}$ with resolvent $\left(1+D_{1}^{*} D_{1}\right)^{-1} \widehat{\otimes} 1 \in$ $\mathbb{L}\left(E_{1} \widehat{\otimes}_{\phi_{2}} E_{2}\right)$.

We now introduce the analogues for the above connection and positivity condition for half-closed chains. They will be shown in Theorem 34 below to indeed correspond to the above two conditions for Kasparov modules.

Definition 27. Given three even half-closed chains $\left(\mathscr{A}, E_{1}, D_{1}\right)$, $\left(\mathscr{B}, E_{2}, D_{2}\right)$ and $\left(\mathscr{A}, E_{1} \widehat{\otimes}_{\phi_{2}} E_{2}, D\right)$ as above, the connection condition 
demands that there exist a dense $\mathscr{B}$-submodule $\mathscr{E}_{1} \subseteq E_{1}$ and cores $\mathscr{E}_{2}$ and $\mathscr{E}$ for $D_{2}: \operatorname{Dom}\left(D_{2}\right) \rightarrow E_{2}$ and $D: \operatorname{Dom}(D) \rightarrow E$, respectively, such that

(a) For each $\xi \in \mathscr{E}_{1}$ :

$$
T_{\xi}\left(\mathscr{E}_{2}\right) \subseteq \operatorname{Dom}(D), \quad T_{\xi}^{*}(\mathscr{E}) \subseteq \operatorname{Dom}\left(D_{2}\right), \quad \gamma_{1}(\xi) \in \mathscr{E}_{1}
$$

(b) For each homogeneous $\xi \in \mathscr{E}_{1}$, the graded commutator

$$
D T_{\xi}-(-1)^{\partial \xi} T_{\xi} D_{2}: \mathscr{E}_{2} \rightarrow E
$$

extends to a bounded operator $L_{\xi}: E_{2} \rightarrow E$.

Definition 28. Given three even half-closed chains $\left(\mathscr{A}, E_{1}, D_{1}\right)$, $\left(\mathscr{B}, E_{2}, D_{2}\right)$ and $\left(\mathscr{A}, E_{1} \widehat{\otimes}_{\phi_{2}} E_{2}, D\right)$ as above, a localizing subset is a countable subset $\Lambda \subseteq \mathscr{A}$ with $\Lambda=\Lambda^{*}$ such that

(a) The subspace

$$
\Lambda \cdot A:=\operatorname{span}_{\mathbb{C}}\{x \cdot a \mid x \in \Lambda, a \in A\} \subseteq A
$$

is norm-dense.

(b) The commutator

$$
\left[D_{1} \widehat{\otimes} 1, \phi(x)\right]: \operatorname{Dom}\left(D_{1} \widehat{\otimes} 1\right) \rightarrow E
$$

is trivial for all $x \in \Lambda$.

(c) We have the domain inclusion

$$
\operatorname{Dom}(D) \cap \operatorname{Im}\left(\phi\left(x^{*} x\right)\right) \subseteq \operatorname{Dom}\left(D_{1} \widehat{\otimes} 1\right),
$$

for all $x \in \Lambda$.

Definition 29. Given three even half-closed chains $\left(\mathscr{A}, E_{1}, D_{1}\right)$, $\left(\mathscr{B}, E_{2}, D_{2}\right)$ and $\left(\mathscr{A}, E_{1} \widehat{\otimes}_{\phi_{2}} E_{2}, D\right)$ and a localizing subset $\Lambda \subseteq \mathscr{A}$, the local positivity condition requires that for each $x \in \Lambda$, there exists a constant $\kappa_{x}>0$ such that

$$
\begin{aligned}
& \left\langle\left(D_{1} \widehat{\otimes} 1\right) \phi\left(x^{*}\right) \xi, D \phi\left(x^{*}\right) \xi\right\rangle+\left\langle D \phi\left(x^{*}\right) \xi,\left(D_{1} \widehat{\otimes} 1\right) \phi\left(x^{*}\right) \xi\right\rangle \\
& \geq-\kappa_{x} \cdot\langle\xi, \xi\rangle,
\end{aligned}
$$

for all $\xi \in \operatorname{Im}(\phi(x)) \cap \operatorname{Dom}\left(D \phi\left(x^{*}\right)\right)$.

Note that the local positivity condition makes sense because of $(\mathrm{d})$ in Definition 28. Indeed, for each $\xi \in \operatorname{Im}(\phi(x)) \cap \operatorname{Dom}\left(D \phi\left(x^{*}\right)\right)$ we have that

$$
\phi\left(x^{*}\right) \xi \in \operatorname{Im}\left(\phi\left(x^{*} x\right)\right) \cap \operatorname{Dom}(D) \subseteq \operatorname{Dom}\left(D_{1} \widehat{\otimes} 1\right) .
$$

Remark 30. Suppose that $\mathscr{A} \subseteq A$ is unital and that $\phi_{1}(A) \cdot E_{1} \subseteq E_{1}$ is norm-dense. Then the half-closed chains $(\mathscr{A}, E, D)$ and $\left(\mathscr{A}, E_{1}, D_{1}\right)$ are in fact unbounded Kasparov modules (thus $D=D^{*}$ and $D_{1}=D_{1}^{*}$ ). The choice $\Lambda:=\{1\} \subseteq \mathscr{A}$ automatically satisfies the conditions (a) and (b) for a localizing subset in Definition 28 and the last condition (c) amounts to the requirement

$$
\operatorname{Dom}(D) \subseteq \operatorname{Dom}\left(D_{1} \widehat{\otimes} 1\right) .
$$


Moreover, in this case, the local positivity condition in Definition 29 means that there exists a constant $\kappa>0$ such that

$$
\left\langle\left(D_{1} \widehat{\otimes} 1\right) \xi, D \xi\right\rangle+\left\langle D \xi,\left(D_{1} \widehat{\otimes} 1\right) \xi\right\rangle \geq-\kappa \cdot\langle\xi, \xi\rangle,
$$

for all $\xi \in \operatorname{Dom}(D)$. Finally, the connection condition in Definition 27 can be seen to be equivalent to the connection condition applied by Kucerovsky in [KUC97]. In this setting, we therefore recover the assumptions applied by Kucerovsky in [KUC97, Theorem 13] (except that the domain condition in [KUC97, Theorem 13] is marginally more flexible). The corresponding special case of Theorem 34 here below, is therefore in itself an improvement to [KUC97, Theorem 13] because of the extra flexibility in the choice of localizing subset $\Lambda \subseteq \mathscr{A}$ (if one is willing to disregard the minor domain issue mentioned earlier in this remark).

We record the following convenient lemma, which can be proved by standard techniques:

Lemma 31. Suppose that the connection condition of Definition 27 holds. Then the connection condition holds for $\mathscr{E}_{2}:=\operatorname{Dom}\left(D_{2}\right)$ and $\mathscr{E}:=\operatorname{Dom}(D)$. Moreover, $L_{\xi}: E_{2} \rightarrow E$ is adjointable with

$$
\left(L_{\xi}\right)^{*}(\eta)=\left(T_{\xi}^{*} D-(-1)^{\partial \xi} D_{2} T_{\xi}^{*}\right)(\eta) \quad \forall \eta \in \operatorname{Dom}(D)
$$

whenever $\xi \in \mathscr{E}_{1}$ is homogeneous.

The next lemma provides a convenient sufficient condition for verifying the inequality in Definition 29:

Lemma 32. Let $x \in A$ and suppose that $\operatorname{Im}\left(\phi\left(x^{*} x\right)\right) \cap \operatorname{Dom}(D) \subseteq$ $\operatorname{Dom}\left(D_{1} \widehat{\otimes} 1\right)$ and that there exists a constant $\kappa_{x}>0$ such that

$$
\left\langle\left(D_{1} \widehat{\otimes} 1\right) \eta, D \eta\right\rangle+\left\langle D \eta,\left(D_{1} \widehat{\otimes} 1\right) \eta\right\rangle \geq-\kappa_{x}\langle\eta, \eta\rangle,
$$

for all $\eta \in \operatorname{Im}\left(\phi\left(x^{*} x\right)\right) \cap \operatorname{Dom}(D)$. Then we have that

$$
\begin{aligned}
& \left\langle\left(D_{1} \widehat{\otimes} 1\right) \phi\left(x^{*}\right) \xi, D \phi\left(x^{*}\right) \xi\right\rangle+\left\langle D \phi\left(x^{*}\right) \xi,\left(D_{1} \widehat{\otimes} 1\right) \phi\left(x^{*}\right) \xi\right\rangle \\
& \quad \geq-\|\phi(x)\|^{2} \kappa_{x}\langle\xi, \xi\rangle,
\end{aligned}
$$

for all $\xi \in \operatorname{Im}(\phi(x)) \cap \operatorname{Dom}\left(D \phi\left(x^{*}\right)\right)$.

Proof. This follows immediately since

$$
-\kappa_{x}\left\langle\phi\left(x^{*}\right) \xi, \phi\left(x^{*}\right) \xi\right\rangle \geq-\|\phi(x)\|^{2} \kappa_{x}\langle\xi, \xi\rangle \quad \forall \xi \in E .
$$

The next lemma is straightforward to prove by rescaling the elements in $\Lambda$ by elements in $(0, \infty)$. It will nonetheless play a very important role:

Lemma 33. Suppose that the local positivity condition of Definition 29 holds with localizing subset $\Lambda \subseteq \mathscr{A}$. Then we may rescale the elements 
in $\Lambda$ and obtain a localizing subset $\Lambda^{\prime} \subseteq \mathscr{A}$ such that the local positivity condition of Definition 29 holds with the additional requirement that

$$
\kappa_{x}=1 / 4 \quad \text { and } \quad\left\|d\left(x^{*}\right) \phi(x)\right\|_{\infty}<1 \quad \forall x \in \Lambda^{\prime} .
$$

Theorem 34. Suppose that the three even half-closed chains $\left(\mathscr{A}, E_{1}, D_{1}\right)$, $\left(\mathscr{B}, E_{2}, D_{2}\right)$ and $\left(\mathscr{A}, E_{1} \widehat{\otimes}_{\phi_{2}} E_{2}, D\right)$ satisfy the connection condition and the local positivity condition. Then $\left(E, F_{D}\right)$ is the Kasparov product of $\left(E_{1}, F_{D_{1}}\right)$ and $\left(E_{2}, F_{D_{2}}\right)$. In particular we have the identity

$$
\left[E, F_{D}\right]=\left[E_{1}, F_{D_{1}}\right] \widehat{\otimes}_{B}\left[E_{2}, F_{D_{2}}\right]
$$

in the KK-group $K K_{0}(A, C)$.

Proof. Without loss of generality we may assume that $\kappa_{x}=1 / 4$ and that $\left\|d\left(x^{*}\right) \phi(x)\right\|_{\infty}<1$ for all $x \in \Lambda$.

We need to prove the connection condition in Equation $(7.1)$ and the positivity condition in Equation $(7.2)$ for the even Kasparov modules $\left(E, F_{D}\right),\left(E_{1}, F_{D_{1}}\right)$ and $\left(E_{2}, F_{D_{2}}\right)$.

But these two conditions are proved in Proposition 35 and Proposition 43 below, respectively. The positivity condition will be satisfied with $\nu=1=4 \cdot \kappa_{x}$.

7.1. The connection condition. We continue working in the setting explained in the beginning of Section 7 .

Before proving our first proposition on the connection condition in Equation (7.1), it will be convenient to introduce some extra notation. For $\lambda \in[0, \infty)$, define the bounded adjointable operators

$$
\begin{aligned}
R(\lambda) & :=\left(1+\lambda+D^{*} D\right)^{-1}, \bar{R}(\lambda):=\left(1+\lambda+D D^{*}\right)^{-1}: E \rightarrow E \\
R_{2}(\lambda) & :=\left(1+\lambda+D_{2}^{*} D_{2}\right)^{-1}, \overline{R_{2}}(\lambda):=\left(1+\lambda+D_{2} D_{2}^{*}\right)^{-1}: E_{2} \rightarrow E_{2} .
\end{aligned}
$$

Proposition 35. Suppose that the connection condition of Definition 27 holds. Then we have that

$$
F_{D} T_{\xi}-(-1)^{\partial \xi} T_{\xi} F_{D_{2}}, F_{D}^{*} T_{\xi}-(-1)^{\partial \xi} T_{\xi} F_{D_{2}}^{*} \in \mathbb{K}\left(E_{2}, E\right),
$$

for all homogeneous $\xi \in E_{1}$.

Proof. Without loss of generality we may assume that $\xi=\eta \cdot b_{1} b_{2}$ with $\eta \in \mathscr{E}_{1}$ homogeneous and $b_{1}, b_{2} \in \mathscr{B}$. Using Lemma 31 we compute as follows, for each $\lambda \in[0, \infty)$ :

$$
\begin{gathered}
R(\lambda) T_{\eta \cdot b_{1}}-T_{\eta \cdot b_{1}} R_{2}(\lambda)=R(\lambda) T_{\eta \cdot b_{1}} D_{2}^{*} D_{2} R_{2}(\lambda)-D^{*} D R(\lambda) T_{\eta \cdot b_{1}} R_{2}(\lambda) \\
=-R(\lambda) T_{\eta} \cdot d_{2}\left(b_{1}\right) \cdot D_{2} R_{2}(\lambda)-(-1)^{\partial \eta} R(\lambda) L_{\eta} \cdot \phi_{2}\left(b_{1}\right) \cdot D_{2} R_{2}(\lambda) \\
\quad+(-1)^{\partial \eta} R(\lambda) D T_{\eta \cdot b_{1}} \cdot D_{2} R_{2}(\lambda)-D^{*} D R(\lambda) T_{\eta \cdot b_{1}} R_{2}(\lambda) \\
=-R(\lambda)\left(T_{\eta} \cdot d_{2}\left(b_{1}\right)+(-1)^{\partial \eta} L_{\eta} \cdot \phi_{2}\left(b_{1}\right)\right) \cdot D_{2} R_{2}(\lambda) \\
-D^{*} \bar{R}(\lambda) L_{\eta \cdot b_{1}} R_{2}(\lambda),
\end{gathered}
$$


where $d_{2}\left(b_{1}\right): E_{2} \rightarrow E_{2}$ is the bounded extension of the commutator $D_{2} \phi_{2}\left(b_{1}\right)-\phi_{2}\left(b_{1}\right) D_{2}^{*}: \operatorname{Dom}\left(D_{2}^{*}\right) \rightarrow E$. In particular, we may find a constant $C>0$ such that

$$
\left\|D R(\lambda) T_{\eta \cdot b_{1}}-D T_{\eta \cdot b_{1}} R_{2}(\lambda)\right\|_{\infty} \leq C \cdot(1+\lambda)^{-1},
$$

for all $\lambda \geq 0$.

We now use the integral formulae

$$
\begin{aligned}
F_{D} & =\frac{1}{\pi} D \cdot \int_{0}^{\infty} \lambda^{-1 / 2} R(\lambda) d \lambda \\
F_{D_{2}} & =\frac{1}{\pi} D_{2} \cdot \int_{0}^{\infty} \lambda^{-1 / 2} R_{2}(\lambda) d \lambda
\end{aligned}
$$

for the bounded transforms. Indeed, using Lemma 31 one more time, these formulae allow us to compute that

$$
\begin{aligned}
F_{D} T_{\xi}= & F_{D} T_{\eta \cdot b_{1}} \cdot \phi_{2}\left(b_{2}\right) \\
= & \frac{1}{\pi} D \cdot T_{\eta \cdot b_{1}} \cdot \int_{0}^{\infty} \lambda^{-1 / 2} R_{2}(\lambda) \cdot \phi_{2}\left(b_{2}\right) d \lambda \\
& \quad+\frac{1}{\pi} D \cdot \int_{0}^{\infty} \lambda^{-1 / 2}\left(R(\lambda) T_{\eta \cdot b_{1}}-T_{\eta \cdot b_{1}} R_{2}(\lambda)\right) \cdot \phi_{2}\left(b_{2}\right) d \lambda \\
= & (-1)^{\partial \xi} T_{\eta \cdot b_{1}} F_{D_{2}} \cdot \phi_{2}\left(b_{2}\right)+\frac{1}{\pi} \int_{0}^{\infty} \lambda^{-1 / 2} L_{\eta \cdot b_{1}} \cdot R_{2}(\lambda) \cdot \phi_{2}\left(b_{2}\right) d \lambda \\
& \quad+\frac{1}{\pi} \int_{0}^{\infty} \lambda^{-1 / 2} D \cdot\left(R(\lambda) T_{\eta \cdot b_{1}}-T_{\eta \cdot b_{1}} R_{2}(\lambda)\right) \cdot \phi_{2}\left(b_{2}\right) d \lambda .
\end{aligned}
$$

The fact that $D_{2} R_{2}(\lambda) \phi_{2}\left(b_{2}\right)$ and $R_{2}(\lambda) \phi_{2}\left(b_{2}\right) \in \mathbb{K}\left(E_{2}\right)$, for all $\lambda \in$ $[0, \infty)$, combined with the estimate in Equation (7.3) now imply that both of the integrals on the right hand side of Equation (7.4) converge absolutely to elements in $\mathbb{K}\left(E_{2}, E\right)$ (remark that the integrands also depend continuously on $\lambda \in(0, \infty)$ with respect to the operator norm). We thus conclude that

$$
F_{D} T_{\xi}-(-1)^{\partial \xi} T_{\eta \cdot b_{1}} F_{D_{2}} \cdot \phi_{2}\left(b_{2}\right) \in \mathbb{K}\left(E_{2}, E\right) .
$$

Since $\left[F_{D_{2}}, \phi_{2}\left(b_{2}\right)\right] \in \mathbb{K}\left(E_{2}\right)$ we have proved that $F_{D} T_{\xi}-(-1)^{\partial \xi} T_{\xi} F_{D_{2}} \in$ $\mathbb{K}\left(E_{2}, E\right)$.

A similar argument shows that $F_{D}^{*} T_{\xi}-(-1)^{\partial \xi} T_{\xi} F_{D_{2}}^{*} \in \mathbb{K}\left(E_{2}, E\right)$ as well.

7.2. Localization. Throughout this subsection the conditions stated in the beginning of Section 7 are in effect.

We are now going to apply the localization results obtained in Section 4. 5 and 6. Recall from Definition 9 and Proposition 11 that whenever $x \in \mathscr{A}$, then the localization $D_{x}: \operatorname{Dom}\left(D_{x}\right) \rightarrow E_{x}$ is the selfadjoint and regular unbounded operator defined as the closure of

$$
\phi(x) D \phi\left(x^{*}\right): \operatorname{Dom}(D) \cap E_{x} \rightarrow E_{x},
$$


where $E_{x}:=\operatorname{cl}(\operatorname{Im}(\phi(x))) \subseteq E$. The core idea is to replace the bounded transform of $D: \operatorname{Dom}(D) \rightarrow E$ by the bounded transforms of sufficiently many localizations $D_{x}: \operatorname{Dom}\left(D_{x}\right) \rightarrow E_{x}$, when verifying the positivity condition in Equation $(7.2)$. The precise result is given here:

Proposition 36. Suppose that conditions (a) and (b) of Definition 28 hold for some localizing subset $\Lambda \subseteq \mathscr{A}$ and that $\nu \in \mathbb{R}$ is given. Suppose moreover that

$$
T_{x}^{*}\left(\left.\left(F_{D_{1}}^{*} \widehat{\otimes} 1\right)\right|_{E_{x}} \cdot F_{D_{x}}+\left.F_{D_{x}} \cdot\left(F_{D_{1}} \widehat{\otimes} 1\right)\right|_{E_{x}}\right) T_{x}+\nu \cdot \phi\left(x^{*} x\right)
$$

is positive in $\mathbb{L}(E) / \mathbb{K}(E)$ for all $x \in \Lambda$. Then we have that

$$
\phi\left(a^{*}\right)\left(\left(F_{D_{1}}^{*} \widehat{\otimes} 1\right) F_{D}^{*}+F_{D}\left(F_{D_{1}} \widehat{\otimes} 1\right)\right) \phi(a)+\nu \cdot \phi\left(a^{*} a\right)
$$

is positive in $\mathbb{L}(E) / \mathbb{K}(E)$ for all $a \in A$.

Proof. For $x \in \Lambda$ we have that $\left[F_{D_{1}} \widehat{\otimes} 1, \phi(x)\right]=0$ and the closed submodule $E_{x} \subseteq E$ is thus invariant under $F_{D_{1}} \widehat{\otimes} 1$. The restriction $\left.\left(F_{D_{1}} \widehat{\otimes} 1\right)\right|_{E_{x}}: E_{x} \rightarrow E_{x}$ is therefore a well-defined bounded adjointable operator. The same observation holds for the adjoint $F_{D_{1}}^{*} \widehat{\otimes} 1$.

Since $\Lambda$ is countable we may write the elements in $\Lambda$ as a sequence $\left\{x_{1}, x_{2}, x_{3}, \ldots\right\}$. For each $n \in \mathbb{N}$, we choose a constant

$$
C_{n}>2+\left\|x_{n}\right\|^{2}+\left\|F_{D} T_{x_{n}}^{*}-T_{x_{n}}^{*} F_{D_{x}}\right\|_{\infty} \cdot\left\|x_{n}\right\|
$$

and define the element

$$
\Gamma:=\sum_{n=1}^{\infty} \frac{1}{n^{2} C_{n}} x_{n}^{*} x_{n} \in A,
$$

where the series is absolutely convergent. Since $\Lambda \cdot A \subseteq A$ is norm-dense and $\Lambda=\Lambda^{*}$ we have that

$$
\Gamma \cdot A \subseteq A
$$

is norm-dense as well. It therefore suffices to show that

$$
\Gamma \cdot\left(\left(F_{D_{1}}^{*} \widehat{\otimes} 1\right) F_{D}^{*}+F_{D}\left(F_{D_{1}} \widehat{\otimes} 1\right)\right) \cdot \Gamma+\nu \cdot \phi\left(\Gamma^{2}\right)
$$

is positive in the Calkin algebra $\mathbb{L}(E) / \mathbb{K}(E)$.

We now compute modulo $\mathbb{K}(E)$, using Proposition 26, that $\Gamma$ commutes with $F_{D_{1}} \widehat{\otimes} 1$ and that $\left(F_{D}, E\right)$ is a Kasparov module:

$$
\begin{aligned}
\Gamma \cdot( & \left.\left(F_{D_{1}}^{*} \widehat{\otimes} 1\right) F_{D}^{*}+F_{D}\left(F_{D_{1}} \widehat{\otimes} 1\right)\right) \cdot \Gamma \\
& \sim \Gamma^{1 / 2}\left(\left(F_{D_{1}}^{*} \widehat{\otimes} 1\right) F_{D}+F_{D}\left(F_{D_{1}} \widehat{\otimes} 1\right)\right) \cdot \Gamma^{3 / 2} \\
& =\Gamma^{1 / 2} \sum_{n=1}^{\infty} \frac{1}{n^{2} C_{n}}\left(\left(F_{D_{1}}^{*} \widehat{\otimes} 1\right) F_{D}+F_{D}\left(F_{D_{1}} \widehat{\otimes} 1\right)\right) T_{x_{n}}^{*} T_{x_{n}} \\
& \sim \Gamma^{1 / 2} \sum_{n=1}^{\infty} \frac{1}{n^{2} C_{n}} T_{x_{n}}^{*}\left(\left.\left(F_{D_{1}}^{*} \widehat{\otimes} 1\right)\right|_{E_{x}} F_{D_{x}}+\left.F_{D_{x}}\left(F_{D_{1}} \widehat{\otimes} 1\right)\right|_{E_{x}}\right) T_{x_{n}} \Gamma^{1 / 2} .
\end{aligned}
$$


But this proves the present proposition since

$$
\begin{gathered}
\Gamma^{1 / 2} \sum_{n=1}^{\infty} \frac{1}{n^{2} C_{n}} T_{x_{n}}^{*}\left(\left.\left(F_{D_{1}}^{*} \widehat{\otimes} 1\right)\right|_{E_{x}} F_{D_{x}}+\left.F_{D_{x}}\left(F_{D_{1}} \widehat{\otimes} 1\right)\right|_{E_{x}}\right) T_{x_{n}} \Gamma^{1 / 2} \\
+\nu \phi\left(\Gamma^{2}\right) \\
=\Gamma^{1 / 2} \sum_{n=1}^{\infty} \frac{1}{n^{2} C_{n}}\left(T_{x_{n}}^{*}\left(\left.\left(F_{D_{1}}^{*} \widehat{\otimes} 1\right)\right|_{E_{x}} F_{D_{x}}+\left.F_{D_{x}}\left(F_{D_{1}} \widehat{\otimes} 1\right)\right|_{E_{x}}\right) T_{x_{n}}\right. \\
\left.+\nu T_{x_{n}}^{*} T_{x_{n}}\right) \cdot \Gamma^{1 / 2}
\end{gathered}
$$

is positive in $\mathbb{L}(E) / \mathbb{K}(E)$ by assumption.

7.3. The positivity condition. We remain in the setup described in the beginning of Section 7 .

Before continuing our treatment of the positivity condition in Equation $(7.2)$ we introduce some further notation:

Definition 37. For each $x \in \mathscr{A}$ satisfying condition (c) in Definition 28 we put

$$
\operatorname{Dom}\left(Q_{x}\right):=\operatorname{Dom}\left(D \phi\left(x^{*}\right)\right) \cap \operatorname{Im}(\phi(x))
$$

and define the map $Q_{x}: \operatorname{Dom}\left(Q_{x}\right) \rightarrow C$ by

$$
Q_{x}(\xi):=2 \cdot \operatorname{Re}\left\langle D \phi\left(x^{*}\right) \xi,\left(D_{1} \widehat{\otimes} 1\right) \phi\left(x^{*}\right) \xi\right\rangle,
$$

where $\mathrm{Re}: C \rightarrow C$ takes the real part of an element in the $C^{*}$-algebra $C$.

For each $\lambda \geq 0$ and $x \in \mathscr{A}$ satisfying condition (b) of Definition 28 we define the bounded adjointable operators on $E_{x}$ :

$$
\begin{aligned}
& \left.R_{1}(\lambda)\right|_{E_{x}}:=\left.\left(1+\lambda+\left(D_{1}^{*} \widehat{\otimes} 1\right)\left(D_{1} \widehat{\otimes} 1\right)\right)^{-1}\right|_{E_{x}} \\
& \left.S_{1}(\lambda)\right|_{E_{x}}:=\left.\left(D_{1} \widehat{\otimes} 1\right)\left(1+\lambda+\left(D_{1}^{*} \widehat{\otimes} 1\right)\left(D_{1} \widehat{\otimes} 1\right)\right)^{-1}\right|_{E_{x}} \\
& R_{x}(\lambda):=\left(1+\lambda+D_{x}^{2}\right)^{-1} \quad S_{x}(\lambda):=D_{x}\left(1+\lambda+D_{x}^{2}\right)^{-1} .
\end{aligned}
$$

The next lemma follows by standard functional calculus arguments:

Lemma 38. Suppose that $x \in \mathscr{A}$ satisfies condition (b) of Definition 28. Then the maps $[0, \infty)^{2} \rightarrow \mathbb{L}\left(E_{x}\right)$ defined by

$$
\begin{aligned}
& M_{1}(\lambda, \mu, x):=\left.S_{x}(\lambda) S_{1}(\mu)\right|_{E_{x}} \\
& M_{2}(\lambda, \mu, x):=\left.S_{x}(\lambda) R_{1}(\mu)\right|_{E_{x}} \cdot \sqrt{1+\mu} \\
& M_{3}(\lambda, \mu, x):=\left.R_{x}(\lambda) S_{1}(\mu)\right|_{E_{x}} \cdot \sqrt{1+\lambda} \\
& M_{4}(\lambda, \mu, x):=\left.R_{x}(\lambda) R_{1}(\mu)\right|_{E_{x}} \cdot \sqrt{(1+\lambda)(1+\mu)}
\end{aligned}
$$

are all continuous in operator norm and satisfy the estimate

$$
\left\|M_{j}(\lambda, \mu, x)\right\|_{\infty} \leq(1+\lambda)^{-1 / 2} \cdot(1+\mu)^{-1 / 2} \quad j \in\{1,2,3,4\},
$$


for all $\lambda, \mu \in[0, \infty)$. In particular, it holds that the integral

$$
\frac{1}{\pi^{2}} \int_{0}^{\infty} \int_{0}^{\infty}(\lambda \mu)^{-1 / 2} \cdot\left(M_{j}^{*} M_{j}\right)(\lambda, \mu, x) d \lambda d \mu
$$

converges absolutely to a bounded adjointable operator $K_{j}(x) \in \mathbb{L}\left(E_{x}\right)$ with $0 \leq K_{j}(x) \leq 1$ for all $j \in\{1,2,3,4\}$.

In order to ensure that later computations are well-defined we prove the following:

Lemma 39. Suppose that $x \in \mathscr{A}$ satisfies condition (c) of Definition 28 and that $\left\|d\left(x^{*}\right) \phi(x)\right\|_{\infty}<1$. Then

$$
\operatorname{Im}\left(R_{x}(\lambda) T_{x}\right) \subseteq \operatorname{Dom}\left(Q_{x}\right) \quad \text { and } \quad \operatorname{Im}\left(S_{x}(\lambda) T_{x}\right) \subseteq \operatorname{Dom}\left(Q_{x}\right),
$$

for all $\lambda \geq 0$. In particular, if $x \in \mathscr{A}$ moreover satisfies condition (b) of Definition 28, then

$$
\operatorname{Im}\left(M_{j}(\lambda, \mu, x) T_{x}\right) \subseteq \operatorname{Dom}\left(Q_{x}\right),
$$

for all $j \in\{1,2,3,4\}$ and all $\lambda, \mu \in[0, \infty)$.

Proof. Recall from Lemma 10 and Proposition 11 that

$$
\left(i r+D_{x}\right)^{-1} T_{x}=T_{x}\left(i r+D \phi\left(x^{*} x\right)\right)^{-1},
$$

for all $r \in \mathbb{R}$ with $|r| \geq 1>\left\|d\left(x^{*}\right) \phi(x)\right\|_{\infty}$. We thus see that

$$
\operatorname{Im}\left(\left(i r+D_{x}\right)^{-1} T_{x}\right) \subseteq \operatorname{Im}(\phi(x)) \cap \operatorname{Dom}\left(D \phi\left(x^{*}\right)\right)=\operatorname{Dom}\left(Q_{x}\right) .
$$

The inclusions in Equation (7.5) now follow since

$$
R_{x}(\lambda) T_{x}=\left(-i \sqrt{1+\lambda}+D_{x}\right)^{-1}\left(i \sqrt{1+\lambda}+D_{x}\right)^{-1} T_{x}
$$

and since

$$
S_{x}(\lambda) T_{x}=D_{x} R_{x}(\lambda) T_{x}=\left(i \sqrt{1+\lambda}+D_{x}\right)^{-1} T_{x}+i \sqrt{1+\lambda} \cdot R_{x}(\lambda) T_{x},
$$

for all $\lambda \geq 0$.

We now start a more detailed computation of the application $Q_{x}$ : $\operatorname{Dom}\left(Q_{x}\right) \rightarrow C$ from Definition 37 .

Lemma 40. Suppose that $x \in \mathscr{A}$ satisfies condition (b) and (c) of Definition 28 and that $\left\|d\left(x^{*}\right) \phi(x)\right\|_{\infty}<1$. Then

$$
\begin{aligned}
Q_{x}\left(S_{x}(\lambda) T_{x}(\xi)\right)=2 \cdot \operatorname{Re}\langle & \left.\left(D_{1} \widehat{\otimes} 1\right) \phi(x) \xi, S_{x}(\lambda) T_{x} \xi\right\rangle \\
& -(1+\lambda) Q_{x}\left(R_{x}(\lambda) T_{x}(\xi)\right),
\end{aligned}
$$

for all $\lambda \in[0, \infty)$ and $\xi \in \operatorname{Dom}\left(\left(D_{1} \widehat{\otimes} 1\right) \phi(x)\right)$.

Proof. Let $\lambda \in[0, \infty)$ and let $\xi \in \operatorname{Dom}\left(\left(D_{1} \widehat{\otimes} 1\right) \phi(x)\right)$ be given. We first claim that

$$
D T_{x}^{*} S_{x}(\lambda) T_{x} \xi \in \operatorname{Dom}\left(\left(D_{1} \widehat{\otimes} 1\right) \phi\left(x^{*} x\right)\right)
$$


and that

$$
\begin{aligned}
& \left(D_{1} \widehat{\otimes} 1\right) \phi\left(x^{*} x\right) D T_{x}^{*} S_{x}(\lambda) T_{x} \xi \\
& \quad=\left(D_{1} \widehat{\otimes} 1\right) \phi\left(x^{*} x\right) \xi-(1+\lambda)\left(D_{1} \widehat{\otimes} 1\right) T_{x}^{*} R_{x}(\lambda) T_{x} \xi
\end{aligned}
$$

But this follows since

$$
\begin{aligned}
& \phi\left(x^{*} x\right) D T_{x}^{*} S_{x}(\lambda) T_{x} \xi=T_{x}^{*} D_{x} S_{x}(\lambda) T_{x} \xi \\
& =\phi\left(x^{*} x\right) \xi-(1+\lambda) T_{x}^{*} R_{x}(\lambda) T_{x} \xi \in \operatorname{Dom}\left(D_{1} \widehat{\otimes} 1\right),
\end{aligned}
$$

where we remark that $\phi\left(x^{*} x\right) \xi \in \operatorname{Dom}\left(D_{1} \widehat{\otimes} 1\right)$ since $x^{*} \in \mathscr{A}$ and that $T_{x}^{*} R_{x}(\lambda) T_{x} \xi \in \operatorname{Dom}(D) \cap \operatorname{Dom}\left(D_{1} \widehat{\otimes} 1\right)$ by condition (c) and Lemma 39 .

Notice now that condition (b) and Proposition 7 implies that

$$
\left(D_{1} \widehat{\otimes} 1\right) \phi\left(x^{*} x\right): \operatorname{Dom}\left(\left(D_{1} \widehat{\otimes} 1\right) \phi\left(x^{*} x\right)\right) \rightarrow E
$$

is selfadjoint and regular. Putting $\eta:=T_{x}^{*} R_{x}(\lambda) T_{x}(\xi) \in \operatorname{Dom}(D) \cap$ $\operatorname{Dom}\left(D_{1} \widehat{\otimes} 1\right)$ and using the above claim, the lemma is then proved by the following computation:

$$
\begin{aligned}
\frac{1}{2} \cdot Q_{x} & \left(S_{x}(\lambda) T_{x}(\xi)\right)=\operatorname{Re}\left\langle D T_{x}^{*} S_{x}(\lambda) T_{x}(\xi),\left(D_{1} \widehat{\otimes} 1\right) T_{x}^{*} S_{x}(\lambda) T_{x}(\xi)\right\rangle \\
\quad= & \operatorname{Re}\left\langle\left(D_{1} \widehat{\otimes} 1\right) \phi\left(x^{*} x\right) D T_{x}^{*} S_{x}(\lambda) T_{x} \xi, D \eta\right\rangle \\
\quad= & \operatorname{Re}\left\langle\left(D_{1} \widehat{\otimes} 1\right) \phi\left(x^{*} x\right) \xi, D \eta\right\rangle-(1+\lambda) \operatorname{Re}\left\langle\left(D_{1} \widehat{\otimes} 1\right) \eta, D \eta\right\rangle \\
\quad= & \operatorname{Re}\left\langle\left(D_{1} \widehat{\otimes} 1\right) \phi(x) \xi, S_{x}(\lambda) T_{x} \xi\right\rangle-(1+\lambda) \operatorname{Re}\left\langle\left(D_{1} \widehat{\otimes} 1\right) \eta, D \eta\right\rangle .
\end{aligned}
$$

Definition 41. For each $x \in \mathscr{A}$ satisfying condition (b) and (c) of Definition 28 and that $\left\|d\left(x^{*}\right) \phi(x)\right\|_{\infty}<1$, we define the assignment

$$
Q_{j}(\lambda, \mu, x): \operatorname{Im}\left(T_{x}\right) \rightarrow C \quad Q_{j}(\lambda, \mu, x)\left(T_{x} \xi\right):=Q_{x}\left(M_{j}(\lambda, \mu, x) T_{x} \xi\right)
$$

for all $\lambda, \mu \in[0, \infty), j \in\{1,2,3,4\}$.

The main algebraic result of this section can now be stated and proved:

Lemma 42. Suppose that $x \in \mathscr{A}$ satisfies condition (b) and (c) of Definition 28 and that $\left\|d\left(x^{*}\right) \phi(x)\right\|_{\infty}<1$. Then we have the identity

$$
\sum_{j=1}^{4} Q_{j}(\lambda, \mu, x)\left(T_{x} \xi\right)=2 \cdot \operatorname{Re}\left\langle T_{x} \xi,\left.S_{x}(\lambda) S_{1}(\mu)\right|_{E_{x}} T_{x} \xi\right\rangle,
$$

for all $\lambda, \mu \in[0, \infty)$ and all $\xi \in E$. 
Proof. Let $\lambda, \mu \in[0, \infty)$ and $\xi \in E$ be given. Remark that $S_{1}(\mu) \xi, R_{1}(\mu) \xi \in$ $\operatorname{Dom}\left(\left(D_{1} \widehat{\otimes} 1\right) \phi(x)\right)$. We may thus use Lemma 40 to compute as follows:

$$
\begin{aligned}
\sum_{j=1}^{4} Q_{j}(\lambda, \mu, x)\left(T_{x} \xi\right) \\
=Q_{x}\left(S_{x}(\lambda) T_{x} S_{1}(\mu) \xi\right)+Q_{x}\left(S_{x}(\lambda) T_{x} R_{1}(\mu) \xi\right)(1+\mu) \\
\quad+Q_{x}\left(R_{x}(\lambda) T_{x} S_{1}(\mu) \xi\right)(1+\lambda) \\
\quad+Q_{x}\left(R_{x}(\lambda) T_{x} R_{1}(\mu) \xi\right)(1+\lambda)(1+\mu) \\
=2 \cdot \operatorname{Re}\left\langle\left(D_{1} \widehat{\otimes} 1\right) \phi(x) S_{1}(\mu) \xi, S_{x}(\lambda) T_{x} S_{1}(\mu) \xi\right\rangle \\
\quad+2 \cdot \operatorname{Re}\left\langle\left(D_{1} \widehat{\otimes} 1\right) \phi(x) R_{1}(\mu) \xi, S_{x}(\lambda) T_{x} R_{1}(\mu) \xi\right\rangle \cdot(1+\mu) \\
=2 \cdot \operatorname{Re}\left\langle T_{x}\left(D_{1}^{*} \widehat{\otimes} 1\right) S_{1}(\mu) \xi, S_{x}(\lambda) T_{x} S_{1}(\mu) \xi\right\rangle \\
\quad+2 \cdot \operatorname{Re}\left\langle T_{x} S_{1}(\mu) \xi, S_{x}(\lambda) T_{x} R_{1}(\mu) \xi\right\rangle \cdot(1+\mu) \\
=2 \cdot \operatorname{Re}\left\langle T_{x} \xi,\left.S_{x}(\lambda) S_{1}(\mu)\right|_{E_{x}} T_{x} \xi\right\rangle
\end{aligned}
$$

This proves the present lemma.

We are now ready to treat the positivity condition in Equation 7.2 :

Proposition 43. Suppose that $\Lambda \subseteq \mathscr{A}$ is a localizing subset satisfying the local positivity condition, that $\left\|d\left(x^{*}\right) \phi(x)\right\|_{\infty}<1$ for all $x \in \Lambda$ and that there exists $a \kappa>0$ such that $\kappa_{x} \leq \kappa$ for all $x \in \Lambda$. Then the inequality

$$
\phi(a)^{*}\left(\left(F_{D_{1}}^{*} \widehat{\otimes} 1\right) F_{D}^{*}+F_{D}\left(F_{D_{1}} \widehat{\otimes} 1\right)\right) \phi(a) \geq-4 \kappa \cdot \phi\left(a^{*} a\right)
$$

holds in the quotient $C^{*}$-algebra $\mathbb{L}(E) / \mathbb{K}(E)$ for all $a \in A$.

Proof. By Proposition 36, it suffices to show that

$$
T_{x}^{*}\left(\left.\left(F_{D_{1}}^{*} \widehat{\otimes} 1\right)\right|_{E_{x}} F_{D_{x}}+\left.F_{D_{x}}\left(F_{D_{1}} \widehat{\otimes} 1\right)\right|_{E_{x}}\right) T_{x}+4 \kappa \phi\left(x^{*} x\right)
$$

is positive in $\mathbb{L}(E) / \mathbb{K}(E)$ for all $x \in \Lambda$. Let thus $x \in \Lambda$ be fixed. We will prove the inequality

$$
2 \cdot \operatorname{Re}\left\langle\left. F_{D_{x}}\left(F_{D_{1}} \widehat{\otimes} 1\right)\right|_{E_{x}} T_{x} \xi, T_{x} \xi\right\rangle \geq-4 \kappa\left\langle T_{x} \xi, T_{x} \xi\right\rangle
$$

in the $C^{*}$-algebra $C$, for all $\xi \in \operatorname{Dom}(D) \cap \operatorname{Dom}\left(D_{1} \widehat{\otimes} 1\right)$. Remark that this is enough since $\operatorname{Dom}(D) \cap \operatorname{Dom}\left(D_{1} \widehat{\otimes} 1\right) \subseteq E$ is norm-dense.

Let thus $\xi \in \operatorname{Dom}(D) \cap \operatorname{Dom}\left(D_{1} \widehat{\otimes} 1\right)$ be given. We have that

$$
\begin{aligned}
2 \cdot \operatorname{Re} & \left.\left\langle\left. F_{D_{x}}\left(F_{D_{1}} \widehat{\otimes} 1\right)\right|_{E_{x}}\right) T_{x} \xi, T_{x} \xi\right\rangle \\
& =\frac{2}{\pi^{2}} \int_{0}^{\infty} \int_{0}^{\infty}(\lambda \mu)^{-1 / 2} \cdot \operatorname{Re}\left\langle\left. S_{x}(\lambda) S_{1}(\mu)\right|_{E_{x}} T_{x} \xi, T_{x} \xi\right\rangle d \lambda d \mu,
\end{aligned}
$$

where the integral converges absolutely in the norm on $C$ and the integrand is norm-continuous from $[0, \infty)^{2}$ to $C$. Now, by Lemma 42 
and the local positivity condition we have that

$$
\begin{gathered}
2 \cdot \operatorname{Re}\left\langle\left. S_{x}(\lambda) S_{1}(\mu)\right|_{E_{x}} T_{x} \xi, T_{x} \xi\right\rangle=\sum_{j=1}^{4} Q_{j}(\lambda, \mu, x)\left(T_{x} \xi\right) \\
\geq-\kappa \cdot \sum_{j=1}^{4}\left\langle M_{j}(\lambda, \mu, x) T_{x} \xi, M_{j}(\lambda, \mu, x) T_{x} \xi\right\rangle .
\end{gathered}
$$

It therefore follows by Lemma 38 that

$$
\begin{aligned}
\frac{2}{\pi^{2}} \int_{0}^{\infty} \int_{0}^{\infty}(\lambda \mu)^{-1 / 2} \cdot \operatorname{Re}\left\langle\left. S_{x}(\lambda) S_{1}(\mu)\right|_{E_{x}} T_{x} \xi, T_{x} \xi\right\rangle \\
\geq-\kappa \cdot \frac{1}{\pi^{2}} \int_{0}^{\infty} \int_{0}^{\infty}(\lambda \mu)^{-1 / 2} \\
\cdot \sum_{j=1}^{4}\left\langle M_{j}(\lambda, \mu, x) T_{x} \xi, M_{j}(\lambda, \mu, x) T_{x} \xi\right\rangle d \lambda d \mu \\
=-\kappa \cdot \sum_{j=1}^{4}\left\langle T_{x} \xi, K_{j}(x) T_{x} \xi\right\rangle \geq-4 \kappa\left\langle T_{x} \xi, T_{x} \xi\right\rangle .
\end{aligned}
$$

But this proves the proposition.

\section{REFERENCES}

[BAJu83] S. BAAJ and P. JuLG, Théorie bivariante de Kasparov et opérateurs non bornés dans les $C^{*}$-modules hilbertiens, C. R. Acad. Sci. Paris Sér. I Math. 296 (1983), no. 21, 875-878. MR 715325 (84m:46091)

[BDT89] P. Baum, R. G. Douglas, and M. E. TAYlor, Cycles and relative cycles in analytic K-homology, J. Differential Geom. 30 (1989), no. 3, 761-804. MR 1021372

[BMvS16] S. Brain, B. Mesland, and W. D. van Suijlekom, Gauge theory for spectral triples and the unbounded Kasparov product, J. Noncommut. Geom. 10 (2016), no. 1, 135-206. MR 3500818

[CGRS14] A. L. Carey, V. Gayral, A. Rennie, and F. A. Sukochev, Index theory for locally compact noncommutative geometries, Mem. Amer. Math. Soc. 231 (2014), no. 1085, vi+130. MR 3221983

[CoMo08] A. Connes and H. Moscovici, Type III and spectral triples, Traces in number theory, geometry and quantum fields, Aspects Math., E38, Friedr. Vieweg, Wiesbaden, 2008, pp. 57-71.

[Con94] A. Connes, Noncommutative geometry, Academic Press, Inc., San Diego, CA, 1994. MR 1303779 (95j:46063)

[Con96] _ Gravity coupled with matter and the foundation of noncommutative geometry, Comm. Math. Phys. 182 (1996), no. 1, 155-176. MR 1441908

[CoSk84] A. Connes and G. Skandalis, The longitudinal index theorem for foliations, Publ. Res. Inst. Math. Sci. 20 (1984), no. 6, 1139-1183. MR 775126 (87h:58209)

[Hil10] M. Hilsum, Bordism invariance in KK-theory, Math. Scand. 107 (2010), no. 1, 73-89. MR 2679393 
[KAA15] J. KAAD, The unbounded Kasparov product by a differentiable module, arXiv:1509.09063 [math.KT].

[KAA16] Morita invariance of unbounded bivariant KK-theory, arXiv: 1612.08405.

[KAA17] _ Differentiable absorption of Hilbert $C^{*}$-modules, connections, and lifts of unbounded operators, To appear in J. Noncommut. Geom. 11 (2017), 1-32. arXiv:1407.1389 [math.0A]

[KALE13] J. KAAD and M. LESCH, Spectral flow and the unbounded Kasparov product, Adv. Math. 248 (2013), 495-530. MR 3107519

[KAs80] G. G. KASPAROV, The operator $K$-functor and extensions of $C^{*}$ algebras, Izv. Akad. Nauk SSSR Ser. Mat. 44 (1980), no. 3, 571-636, 719. MR 582160 (81m:58075)

[KavS17] J. KaAD and W. D. VAn SuIJlekom, Factorization of Dirac operators on almost-regular fibrations of spin ${ }^{c}$ manifolds, Preprint, 2017.

[Kuc97] D. KuCERovsky, The KK-product of unbounded modules, K-Theory 11 (1997), no. 1, 17-34. MR 1435704 (98k:19007)

[LAn95] E. C. LANCE, Hilbert $C^{*}$-modules, London Mathematical Society Lecture Note Series, vol. 210, Cambridge University Press, Cambridge, 1995, A toolkit for operator algebraists. MR 1325694 (96k:46100)

[LAT13] F. LATRÉmoliÈRE, Quantum locally compact metric spaces, J. Funct. Anal. 264 (2013), no. 1, 362-402. MR 2995712

[MeRe16] B. Mesland and A. Rennie, Nonunital spectral triples and metric completeness in unbounded KK-theory, J. Funct. Anal. 271 (2016), no. 9, 2460-2538. MR 3545223

[Mes14] B. MESLAND, Unbounded bivariant K-theory and correspondences in noncommutative geometry, J. Reine Angew. Math. 691 (2014), 101172. MR 3213549

[Wor91] S. L. WoronowiCz, Unbounded elements affiliated with $C^{*}$-algebras and noncompact quantum groups, Comm. Math. Phys. 136 (1991), no. 2, 399-432. MR 1096123 (92b:46117)

Department of Mathematics and Computer Science, Syddansk Universitet, Campusvej 55, 5230, Odense M, Denmark

E-mail address: jenskaad@hotmail.com

Institute for Mathematics, Astrophysics and Particle Physics, RadBoud University Nijmegen, HeyendaAlseweg 135, 6525 AJ Nijmegen, The Netherlands

E-mail address: waltervs@math.ru.nl 